\title{
Optimal Waveform Design for UWB Radios
}

\author{
Xianren Wu, Student Member, IEEE, Zhi Tian, Member, IEEE, Timothy N. Davidson, Member, IEEE, and \\ Georgios B. Giannakis, Fellow, IEEE
}

\begin{abstract}
With transmit power spectra strictly limited by regulatory spectral masks, the emerging ultra-wideband (UWB) communication systems call for judicious pulse shape design in order to achieve optimal spectrum utilization, spectral mask compatibility, and coexistence with other wireless services. Meanwhile, orthogonal pulse sets are often desired in order to apply high-rate multidimensional modulation and (carrier-free) orthogonal frequency-division multiple access. Motivated by these considerations, we suggest a digital finite impulse response (FIR) filter approach to synthesizing UWB pulses and propose filter design techniques by which optimal waveforms that satisfy the spectral mask can be efficiently obtained. For single pulse design, we develop a convex formulation for the design of the FIR filter coefficients that maximize the spectrum utilization efficiency in terms of both the bandwidth and power allowed by the spectral mask. For orthogonal pulse design, a sequential strategy is derived to formulate the overall pulse design problem as a set of convex subproblems, which are then solved in a sequential manner to yield a set of mutually orthogonal pulses. Our design techniques not only provide waveforms with high spectrum utilization and guaranteed spectral mask compliance but also permit simple modifications that can accommodate several other system objectives.
\end{abstract}

Index Terms-Digital pulse design, finite impulse response (FIR) filter, ultra-wide-band communications.

\section{INTRODUCTION}

W ITH the release of the U.S. Federal Communications Commission (FCC) spectral masks in 2002 [1], ultra-wideband (UWB) radios have attracted great interest for their potential application in short-range high-data-rate wireless communications [19]. With its enormous bandwidth, UWB signaling provides fine temporal resolution and offers the potential for ample multipath diversity. Baseband UWB systems also enable simple transceiver structures with system-on-chip (SoC) implementations. However, the benefits of UWB signaling may be offset by the interference to and from existing systems operating over the same frequency bands. For spectrum overlay

Manuscript received August 27, 2004; revised June 16, 2005. The work of Z. Tian was supported by the National Science Foundation (NSF) under Grant CCR-0238174. The work of T. N. Davidson was supported by NSERC under a Discovery Grant. The work of G. B. Giannakis was supported by ARL/CTA under Grant DAAD19-01-2-011 and by NSF under Grant EIA-0324804. This paper was presented in part at the IEEE ICASSP Conference, Montreal, QC, Canada, May 2004, and the IEEE SPAWC Conference, Lisbon, Portugal, July 2004. The associate editor coordinating the review of this manuscript and approving it for publication was Dr. Peter M. Djuric.

$\mathrm{X}$. Wu and Z. Tian are with the Department of Electrical and Computer Engineering, Michigan Technological University, Houghton, MI 49931 USA (e-mail: xwu@mtu.edu; ztian@mtu.edu).

T. N. Davidson is with the Department of Electrical and Computer Engineering, McMaster University, Hamilton, ON L8S 4K1, Canada (e-mail: davidson@mcmaster.ca).

G. B. Giannakis is with the Department of Electrical and Computer Engineering, University of Minnesota, Minneapolis, MN 55455 USA (e-mail: georgios@ece.umn.edu).

Digital Object Identifier 10.1109/TSP.2006.872556

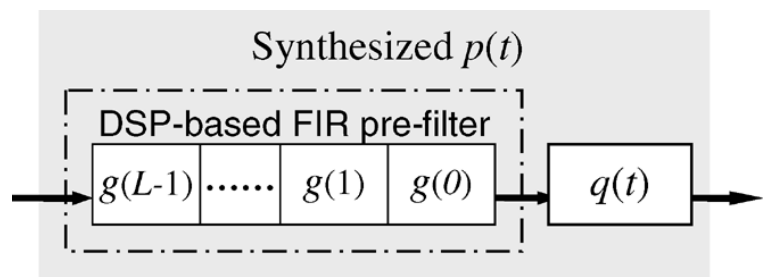

Fig. 1. DSP-based UWB pulse design.

control, the FCC regulations imposed a spectral mask that strictly constrains the transmission power of a UWB signal to be well below the noise floor in all bands. On the other hand, the transmission reliability of a UWB system is determined by the received signal-to-noise ratio (SNR). Given the stringent transmission power limitations, maximization of the received SNR requires efficient utilization of the bandwidth and power allowed by the FCC mask. Since the spectrum of the transmitted signal is effectively determined by that of the underlying UWB pulse, the choice of the pulse shape is a key design decision in UWB systems.

Unfortunately, the widely adopted Gaussian monocycle pulse [20], [25] exhibits a poor fit to the FCC spectral mask and thus is not desirable for practical usage. Recently, [17] proposed that the pulses be based on the dominant eigenvectors of a channel matrix that is constructed by sampling the spectral mask. Pulses generated from different eigenvectors are mutually orthogonal, and conform to the FCC spectral mask. However, they do not achieve the optimal spectral utilization, and require a high sampling rate $(64 \mathrm{GHz})$ that could lead to implementation difficulties. Digital FIR filter solutions based on the Parks-McClellan (PM) algorithm [14] have also been exploited for shaping UWB pulses under mask-fitting requirements [13]. The PM design facilitates good approximations of the FCC spectral mask in a minimax sense but does not directly optimize the spectral utilization of the pulse. Moreover, trial-and-error may be required to find suitable values for the parameters implicit in a PM design, such as the edges tolerances of the pass- and stopbands, and the frequency weighting of the approximation error.

In this paper, we develop optimal design techniques for waveforms synthesized by the digital FIR filter structure proposed in [13]; see also Fig. 1. A convenient basis pulse, such as a Gaussian monocycle, is used as the building block. Prior to modulating this basis pulse, the channel symbols are passed through a linear FIR prefilter, whose filter tap coefficients are carefully designed to generate the desired synthesized pulse. Rather than resorting to the PM algorithm, we seek digital filter designs that maximize the power utilization efficiency while complying with the spectral mask and employ efficient algorithms with well-behaved convergence properties. For the single pulse design, our approach formulates an optimization problem which 
has the spectral utilization as its objective and explicitly imposes all design constraints, including the spectral mask constraint. The autocorrelation properties of a pulse shape are then exploited to precisely transform the initial, nonconvex, optimization problem into a convex problem from which a globally optimal filter can be efficiently obtained using general-purpose convex cone optimization tools, e.g., [22]. The resulting design method yields pulses that maximize the spectral utilization and are guaranteed to conform to the spectral mask, and hence there is no need to interactively search for suitable parameter values (as there is in the PM approach). Achieving optimality also offers the potential for using a lower sampling frequency than competing methods, which may simplify practical hardware realization.

Motivated by the need for high data rates, orthogonal frequency multiplexing, and high spectral efficiency multidimensional modulations, we extend the FIR filter approach to design spectrally efficient orthogonal pulses. The goal is to design a set of mutually orthogonal pulses, each of which occupies the entire spectrum allowed by the spectral mask. Each pulse so designed represents an independent dimension of the signal space. Although they have a digital signal-processing (DSP) implementation similar to that for the single pulses, the design of multiple orthogonal pulses cannot directly borrow the formulation used in single-pulse designs. ${ }^{1}$ However, we still wish to express the design problem in terms of a manageable (preferably convex) objective and constraints on the filter tap coefficients. To do so, we propose a sequential design strategy in which the design of any new pulse is formulated as an FIR filter design problem with linear constraints imposed to ensure that the new pulse is orthogonal to all previously designed pulses. By doing so, a set of $K$ orthogonal pulses can be generated by solving $K$ convex subproblems formulated in a sequential manner. We demonstrate that this sequential filter design approach is able to generate sets of orthogonal pulses that attain large spectrum utilizations under the spectral mask.

The rest of this paper is organized as follows. Section II describes the signal model, problem statement, and FIR filter structure for UWB waveform synthesis. Convex formulations of the single pulse design problem are derived in Section III, and orthogonal pulse design is addressed via a sequential design strategy in Section IV. Some design examples with comparisons to existing algorithms are provided in these two sections. Concluding remarks are provided in Section V.

\section{SIGNAL MODEL}

In a UWB impulse radio, each information symbol is conveyed over a train of $N_{f}$ repeated basic pulses, with one pulse per frame of duration $T_{f}$. Each unit-energy pulse $p(t)$ has an ultrashort duration $T_{p}\left(T_{p} \ll T_{f}\right)$ at the nanosecond scale. The equivalent symbol signature waveform is $p_{s}(t):=\sum_{n=0}^{N_{f}-1} p\left(t-c_{n} T_{c}-n T_{f}\right)$ and has symbol duration $T_{s}:=N_{f} T_{f}$, where the sequence $\left\{c_{n}\right\}_{n=0}^{N_{f}-1}$ represents the user-specific pseudorandom time-hopping (TH) code with

\footnotetext{
${ }^{1}$ The single-pulse designs depend on the power spectrum of the filter, whereas the design of multiple orthogonal pulses with large spectral utilization concerns both the magnitude and phase of the frequency response of the filter.
}

$c_{n} T_{c}<T_{f}, \forall n \in\left[0, N_{f}-1\right]$. Let $b_{k} \in\{ \pm 1\}$ be independent identically distributed binary data symbols with energy $\mathcal{E}_{s}$ spread over $N_{f}$ frames. When pulse amplitude modulation (PAM) is used, the transmitted PAM UWB waveform is given by

$$
u(t)=\sqrt{\frac{\mathcal{E}_{s}}{N_{f}}} \sum_{k} b_{k} p_{s}\left(t-k T_{s}\right) .
$$

The power spectral density (PSD) of $u(t)$ is then given by

$$
\Phi_{u u}(f)=\frac{\mathcal{E}_{s}}{N_{f}} \cdot \frac{1}{T_{s}}\left|P_{s}(f)\right|^{2}
$$

where $P_{s}(f)$ is the Fourier transform (FT) of $p_{s}(t)$ and depends on both $p(t)$ and the TH code $\left\{c_{n}\right\}_{n=0}^{N_{f}-1}$. Specifically, $P_{s}(f)$ can be expressed as

$$
P_{s}(f)=P(f) \sum_{n=0}^{N_{f}-1} e^{-j 2 \pi f n T_{f}} e^{-j 2 \pi f c_{n} T_{c}}
$$

where $P(f)$ is the FT of $p(t)$. Equation (2) now becomes

$$
\Phi_{u u}(f)=\frac{\mathcal{E}_{s}}{T_{s} N_{f}}|P(f)|^{2}\left|\sum_{n=0}^{N_{f}-1} e^{-j 2 \pi f\left(n T_{f}+c_{n} T_{c}\right)}\right|^{2} .
$$

When the TH code $\left\{c_{n}\right\}_{n=0}^{N_{f}-1}$ is independent and uniformly distributed over $\left[0, N_{c}-1\right]$ with integer values, $\Phi_{u u}(f)$ can be approximated as [24]

$$
\Phi_{u u}(f) \approx \alpha|P(f)|^{2}
$$

where $\alpha=\mathcal{E}_{s} / T_{f}$ is a constant. A similar result is also derived in [13] for pulse position modulation (PPM) UWB waveforms. In both cases, a UWB transmitter can be treated as a linear amplifier of the pulse shaper $p(t)$. Hence, the UWB pulse design problem is equivalent to designing the basic pulse $p(t)$ to meet the relevant system requirements.

The FCC regulatory requirements for UWB communication systems state that the power measured across the UWB spectrum must lie below a specified spectral mask. In order to satisfy this requirement, the UWB pulse $p(t)$ should be designed so that $|P(f)|^{2} \leq S_{\mathrm{FCC}}(f)$, where $S_{\mathrm{FCC}}(f)$ is an appropriately scaled version of the regulatory mask. In practice, one typically imposes a tighter spectral mask, say, $S(f)$, on $|P(f)|^{2}$ in the design phase in order to ensure that the regulatory requirements are satisfied in practical implementations, such as those in which nonlinearities in the transmission path cause spectral regrowth. That is, setting $S(f) \leq S_{\mathrm{FCC}}(f)$ for all $f$, we impose

$$
|P(f)|^{2} \leq S(f)
$$

We consider a DSP-based pulse design scheme that builds upon a basis pulse $q(t)$ readily available from a UWB pulse generator. Examples of $q(t)$ include the widely promoted Gaussian monocycle and the sinc pulse. Suppose $q(t)$ is of duration $T_{q}$ and has a Fourier transform $Q(f)$. Our synthesized pulse $p(t)$ to be transmitted over a wireless channel can be written as [13]

$$
p(t)=\sum_{k=0}^{L-1} g[k] q\left(t-k T_{0}\right)
$$


where $T_{0}$ is the sampling interval and the set $\{g[k]\}_{k=0}^{L-1}$ contains the $L$ (real) coefficients to be designed so that (6) is satisfied. The clock rate of the transmitter is $F_{0}:=1 / T_{0}$, and the pulse duration $T_{p}$ of $p(t)$ is $T_{p}=(L-1) T_{0}+T_{q}$. If we view the design parameters $\{g[k]\}$ as the tap coefficients of an $L$-tap FIR filter, our approach to shaping the waveform $p(t)$ reduces to FIR linear prefiltering, as illustrated in Fig. 1; see also [13].

For notational convenience, we will stack the impulse response of $g[k]$ into a vector $\mathbf{g}:=[g[0], g[1], \ldots, g[L-1]]^{T}$. Similarly, the autocorrelation of $g[k]$, namely, $r[m]=$ $\sum_{k} g[k] g[k+m]$, will sometimes be represented by $\tilde{\mathbf{r}}=[r[0], r[1], \ldots, r[L-1]]^{T}$. To concisely describe the frequency components of an $L$-tap FIR filter, we define

$$
\begin{gathered}
\mathbf{v}(f, L)=\left[1, e^{j 2 \pi f T_{0}}, e^{j 2 \pi f 2 T_{0}}, \ldots, e^{j 2 \pi f(L-1) T_{0}}\right]^{T} \\
\tilde{\mathbf{v}}(f, L)=\left[1,2 \cos \left(2 \pi f T_{0}\right), 2 \cos \left(2 \pi f 2 T_{0}\right), \ldots,\right. \\
\left.\ldots, 2 \cos \left(2 \pi f(L-1) T_{0}\right)\right]^{T}
\end{gathered}
$$

where $\mathbf{v}(f, L)$ and $\tilde{\mathbf{v}}(f, L)$ form the basis of complex-phase and linear-phase components, respectively. Consequently, the frequency response of $g[k]$ can be written as $G\left(e^{j 2 \pi f T_{0}}\right)=$ $\sum_{k=0}^{L-1} g[k] e^{-j 2 \pi f k T_{0}}=\mathbf{v}^{H}(f, L) \mathbf{g}$. The power spectrum $S_{p}(f):=|P(f)|^{2}$ of $p(t)$ is $S_{p}(f)=S_{q}(f) S_{q}(f)$, where $S_{q}(f)=|Q(f)|^{2}$ is the power spectrum of $q(t)$ and $S_{g}(f)=\left|G\left(e^{j 2 \pi f T_{0}}\right)\right|^{2}$.

\section{Optimal UWB Single PUlse Design}

In this section, we study various formulations of the single pulse design problem. Our goal is to find a synthesized waveform $p(t)$ that maximizes the spectral utilization efficiency, while at the same time respecting the spectral mask $S(f)$. The spectral utilization efficiency can be measured in terms of the normalized effective signal power (NESP), which is the ratio of the power transmitted in the designated "passband" of the spectral mask over the total power that is permissible under the given mask. Formally stated, if $\mathcal{F}_{p}$ denotes the band (or collection of bands) that constitute the passband, then the NESP is defined as $\bar{\psi}=\int_{\mathcal{F}_{p}} S_{p}(f) d f / \int_{\mathcal{F}_{p}} S(f) d f$. Because $S(f)$ is independent of our design parameters, maximizing $\bar{\psi}$ is equivalent to maximizing

$$
\psi=\int_{\mathcal{F}_{p}} S_{p}(f) d f=\mathbf{g}^{T} \mathbf{Q g}
$$

where $\mathbf{Q}=\int_{\mathcal{F}_{p}} S_{q}(f) \mathbf{v}(f, L) \mathbf{v}^{H}(f, L) d f$. If $S_{q}(f)$ has a sufficiently simple analytic form over the passband, then $\mathbf{Q}$ can be calculated analytically; e.g., when $S_{q}(f)$ is constant over the passband. In other cases, $\mathbf{Q}$ can be computed numerically, even when we only know samples of $S_{q}(f)$ rather than its functional form.

\section{A. Direct Maximization of NESP}

The direct statement of our design problem is as follows.

Problem 1: Given $L, T_{0}, S_{q}(f)$, and $S(f)$, find a filter $g[k]$ of length $L$ that maximizes $\psi$, subject to the spectral mask constraint $S_{p}(f) \leq S(f)$ for all $f$, or show that none exists.
To formulate this problem better, let $\mathbb{R}_{+}$denote the set of nonnegative real numbers, and define the extended function $\breve{M}(f)$ : $\mathbb{R} \rightarrow \mathbb{R}_{+} \bigcup\{\infty\}$ as

$$
\breve{M}(f)=\left\{\begin{array}{ll}
\frac{S(f)}{S_{q}(f)}, & \text { if } S_{q}(f)>0 \\
+\infty, & \text { if } S_{q}(f)=0
\end{array} .\right.
$$

The spectral mask constraint $S_{p}(f) \leq S(f)$ is equivalent to

$$
S_{g}(f) \leq \breve{M}(f) \text { for all } f \text {. }
$$

To establish a more explicit formulation of (12), we define $\mathbf{A}(f)=[\operatorname{Re}(\mathbf{v}(f, L)),-\operatorname{Im}(\mathbf{v}(f, L))]^{T}$, where $\operatorname{Re}(\cdot)$ and $\operatorname{Im}(\cdot)$ denote the real and imaginary parts, respectively. By recognizing that $\sqrt{S_{g}(f)}=\|\mathbf{A}(f) \mathbf{g}\|_{2}$ and defining 2

$$
M(f)=\min _{n \in \mathbb{Z}} \breve{M}\left(f+\frac{n}{T_{0}}\right)
$$

over the domain $f \in\left[0,1 /\left(2 T_{0}\right)\right]$, Problem 1 can be formulated as

$$
\max _{\mathbf{g}} \quad \psi=\mathbf{g}^{T} \mathbf{Q g}
$$

$$
\text { subject to } \quad\|\mathbf{A}(f) \mathbf{g}\|_{2} \leq \sqrt{M(f)} \quad \text { for all } f \in\left[0, \frac{1}{\left(2 T_{0}\right)}\right] \text {. }
$$

The feasible set in (14) is defined by the intersection of an infinite number of second-order cone constraints on linear transformations of $\mathrm{g}$, one for each $f$; see (14b). Hence, it is convex [8]. However, (14b) defines an infinite number of constraints that must be rendered finite. One way to approximate (14b) is to sample it uniformly in frequency and replace it by

$$
\begin{aligned}
\left\|\mathbf{A}\left(f_{n}\right) \mathbf{g}\right\|_{2} \leq \sqrt{M\left(f_{n}\right)}-\epsilon_{N_{\mathrm{d}}} & \\
& \text { for all } f_{n} \in \mathrm{F}^{N_{\mathrm{d}}}:\left\{\frac{n}{\left(2 N_{\mathrm{d}} T_{0}\right)}\right\}_{n=0}^{N_{\mathrm{d}}} .
\end{aligned}
$$

Typically $N_{\mathrm{d}}$ is chosen to be on the order of $15 L$. The parameter $\epsilon_{N_{\mathrm{d}}} \geq 0$ can be chosen such that satisfaction of (15) guarantees satisfaction of (14b) even for $f \notin \mathrm{F}^{N_{\mathrm{d}}}$ (e.g., [26]), or it can be chosen to be a smaller number. If $\epsilon_{N_{\mathrm{d}}}$ is chosen to be zero, then (15) is a direct relaxation of (14b). Depending on the choice of $\epsilon_{N_{\mathrm{d}}}$, the feasible set of the discretized problem will be slightly smaller, or slightly larger, than that of the original problem. Nevertheless, the feasible set remains convex.

Unfortunately, the objective in (14a) is a convex quadratic function of $\mathrm{g}$, and since it is to be maximized under cone constraints, (14) is a nonconvex optimization problem. Therefore, any algorithm for the solution of (14) must be able to deal with the intricacies of locally optimal solutions. ${ }^{3}$ However, both the objective and the constraints in (14) are linear functions of the autocorrelation of $\mathrm{g}$. In particular, by exploiting the fact that $\left|\mathbf{v}^{H}(f, L) \mathbf{g}\right|^{2}=\left|G\left(e^{j 2 \pi f T_{0}}\right)\right|^{2}=R\left(e^{j 2 \pi f T_{0}}\right)=\tilde{\mathbf{v}}^{T}(f, L) \tilde{\mathbf{r}}$, it can be shown that $\psi=\mathbf{c}^{T} \tilde{\mathbf{r}}$, where $\mathbf{c}=\int_{\mathcal{F}_{p}} S_{q}(f) \tilde{\mathbf{v}}(f, L) d f$

\footnotetext{
${ }^{2}$ In many cases we will have $M(f)=\breve{M}(f)$ for all $f \in\left[0,1 /\left(2 T_{0}\right)\right]$.
}

${ }^{3}$ Rigorous methods (e.g., [2]) are effective but tend to be computationally expensive, and simpler techniques, such as running a local optimization routine from multiple starting points, are less expensive but may not be as effective. 
and $\|\mathbf{A}(f) \mathbf{g}\|_{2}^{2}=\tilde{\mathbf{v}}^{T}(f, L) \tilde{\mathbf{r}}$. Therefore, Problem 1 can be reformulated as the following convex optimization problem in the autocorrelation of the filter:4

$$
\begin{aligned}
& \max _{\tilde{\mathbf{r}}} \quad \psi=\mathbf{c}^{T} \tilde{\mathbf{r}} \\
& \text { such that } \quad \tilde{\mathbf{v}}^{T}(f, L) \tilde{\mathbf{r}} \leq M(f) \quad \text { for all } f \in\left[0, \frac{1}{\left(2 T_{0}\right)}\right] \\
& \tilde{\mathbf{v}}^{T}(f, L) \tilde{\mathbf{r}} \geq 0 \quad \text { for all } f \in\left[0, \frac{1}{\left(2 T_{0}\right)}\right]
\end{aligned}
$$

This problem is a semi-infinite linear program (SILP; e.g., [15]) in which there are two constraints for each $f$. The linear constraint set in (16c) is a necessary and sufficient condition for $\tilde{\mathbf{r}}$ to represent a valid autocorrelation sequence. The semi-infinite constraints can be discretized to form a finite linear program whose solution can be efficiently found [15], [16]. While that solution is an approximation to the true solution, discretization strategies exist that ensure this approximation to be a good one; e.g., the direct analogy of (15). As an alternative to discretization, the constraint in (16c) can be precisely enforced using a single linear matrix inequality (LMI) constraint (e.g., [10]), which will result in a (convex) semidefinite programming (SDP; [6], [23]) formulation of the design problem (e.g., [9]-[11]). If $M(f)$ is a piecewise trigonometric polynomial, the mask constraint in (16b) can also be precisely enforced using LMI constraints [4], [10]. Once the optimal autocorrelation has been found, an optimal filter $g[k]$ can be found via spectral factorization; e.g., [12], [26]. A design example of the precise SDP implementation for solving (16) appears in [27].

\section{B. Constrained Power Spectrum Approximation}

An alternative to directly maximizing the NESP is to take an indirect approach in which the objective is to keep the power spectrum of the pulse $S_{p}(f)$ as close to the spectral mask $S(f)$ as possible over the specified passband. While it may appear to be unnecessary at this point, we discuss indirect approaches here because they will provide a method for extending our design technique to the case of multiple orthogonal pulses.

To formalize the notion of closeness, we will use functional norms of the form

$$
\varphi_{\ell}:=\left\|S(f)-S_{p}(f)\right\|_{\mathcal{L}_{\ell}\left(\mathcal{F}_{p}\right)}
$$

where

$$
\|X(f)\|_{\mathcal{L}_{\ell}\left(\mathcal{F}_{p}\right)}=\left\{\begin{array}{ll}
\left(\int_{\mathcal{F}_{p}}|X(f)|^{\ell} d f\right)^{\frac{1}{\ell}} & \text { for } 1 \leq \ell<\infty \\
\max _{f \in \mathcal{F}_{p}}|X(f)| & \text { for } \ell=\infty
\end{array} .\right.
$$

Since $S_{p}(f)$ is real, and since we will constrain it so that $S_{p}(f) \leq S(f)$, we can neglect the absolute-value operator $|\cdot|$ in (18). Using this notation, the constrained power spectrum approximation problem can be written as follow.

Problem 2: Given $\ell, L, T_{0}, S(f)$, and $S_{q}(f)$, find a filter $g[k]$ of length $L$ that achieves

$$
\begin{array}{cl}
\min _{\mathbf{g}} \quad \varphi_{\ell}=\left\|S(f)-S_{p}(f)\right\|_{\mathcal{L}_{\ell}\left(\mathcal{F}_{p}\right)} \\
\text { subject to } \quad S_{p}(f) \leq S(f) \quad \text { for all } f
\end{array}
$$

or show that none exists.

\footnotetext{
${ }^{4}$ Several related pulse design problems can also be cast as convex optimization problems in $\tilde{\mathbf{r}}$; e.g., [9], [11], and references therein.
}

The feasible set defined by (19b) is the same as that in Problem 1. Depending on the choice of $\ell$, different formulations of Problem 2 arise. In particular, since (19b) holds, the unnormalized NESP is $\psi:=-\varphi_{1}+\int_{\mathcal{F}_{p}} S(f) d f$. Therefore, when $\ell=1$, Problem 2 is equivalent to Problem 1, and the formulations in (14) and (16) can be used to solve (19).

Another interesting case arises when the choice $\ell=\infty$ is made. By writing the minimization of an infinity norm in its epigraph form (e.g., [6, p. 293]), we can formulate Problem 2 for $\ell=\infty$ as

$$
\begin{aligned}
& \min _{\mathbf{g}, t} t \\
& \text { such that } \quad\|\mathbf{A}(f) \mathbf{g}\|_{2}^{2} \leq M(f) \quad \text { for all } f \in\left[0, \frac{1}{\left(2 T_{0}\right)}\right] \\
& \|\mathbf{A}(f) \mathbf{g}\|_{2}^{2} \geq \breve{M}(f)-\frac{t}{S_{q}(f)} \quad \text { for all } f \in \mathcal{F}_{p} .
\end{aligned}
$$

Unfortunately, (20c) is a set of nonconvex quadratic constraints, and hence (20) is not convex. However, using the techniques of the previous subsection, the problem in (20) can be recast as the following semi-infinite linear program in $\tilde{\mathbf{r}}$

$$
\begin{aligned}
& \min _{\tilde{\mathbf{r}}, t} \quad t \\
& \text { such that } \quad \tilde{\mathbf{v}}^{T}(f, L) \tilde{\mathbf{r}} \leq M(f) \quad \text { for all } f \in\left[0, \frac{1}{\left(2 T_{0}\right)}\right] \\
& \tilde{\mathbf{v}}^{T}(f, L) \tilde{\mathbf{r}} \geq \breve{M}(f)-\frac{t}{S_{q}(f)} \quad \text { for all } f \in \mathcal{F}_{p} \\
& \tilde{\mathbf{v}}^{T}(f, L) \tilde{\mathbf{r}} \geq 0 \quad \text { for all } f \in\left[0, \frac{1}{\left(2 T_{0}\right)}\right] .
\end{aligned}
$$

Here, (21d) ensures that $\tilde{\mathbf{r}}$ is a valid autocorrelation sequence.

\section{Constrained Frequency Response Approximation}

Problem 2 can be viewed as designing a filter so that the power spectrum $S_{p}(f)$ of the pulse $p(t)$ approximates the mask $S(f)$, but never exceeds it. Such problems typically lead to nonconvex optimization problems in $\mathrm{g}$, but, as we have shown, they can often be precisely transformed into convex problems in $\tilde{\mathbf{r}}$. An alternative approach, which can lead to design problems which are convex in $\mathrm{g}$, is to optimize an $\mathcal{L}_{\ell}\left(\mathcal{F}_{p}\right)$ approximation of the pulse frequency response $P(f)=Q(f) G\left(e^{j 2 \pi f T_{0}}\right)$, rather than its power spectrum. Given that we want the power spectrum to be close to $S(f)$, the desired pulse frequency response is given by $e^{j \theta^{(d)}(f)} \sqrt{S(f)}$, where $\theta^{(d)}(f)$ is a phase component chosen by the designer. The accuracy of the approximation of the frequency response will be measured by

$$
\phi_{\ell}:=\left\|e^{j \theta^{(d)}(f)} \sqrt{S(f)}-Q(f) G\left(e^{j 2 \pi f T_{0}}\right)\right\|_{\mathcal{L}_{\ell}\left(\mathcal{F}_{p}\right)} .
$$

The approximation error in (22) involves both magnitude and phase components of the frequency response, whereas that in (17) involves only the magnitude (squared) components. Filter design problems in the complex domain, e.g., via incorporating the complex domain error in (22), are often convex in $\mathrm{g}$ [7]. One compromise that we have made to obtain convexity in $\mathbf{g}$ is that the design is now dependent on the choice of $\theta^{(d)}(f)$. However, in certain circumstances, such as those in Section III-D below, there are natural choices for $\theta^{(d)}(f)$. We now formalize the design problem: 
Problem 3: Given $\ell, L, T_{0}, S(f), \theta^{(d)}(f)$ and $Q(f)$, find a filter $g[k]$ of length $L$ that achieves

$$
\min _{\mathbf{g}} \quad \phi_{\ell}=\left\|e^{j \theta^{(d)}(f)} \sqrt{S(f)}-Q(f) G\left(e^{j 2 \pi f T_{0}}\right)\right\|_{\mathcal{L}_{\ell}\left(\mathcal{F}_{p}\right)}
$$

such that $S_{p}(f) \leq S(f)$ for all $f$

or show that none exists.

Since the choice $\ell=1$ in Problem 2 is equivalent to Problem 1 , and since the components of the argument of the norm in (22) are "square roots" of the components of the argument of the norm in (17), a natural choice for $\ell$ in Problem 3 is $\ell=2$. For that choice, the objective in Problem 3 can be written as

$$
\phi_{2}=\mathbf{g}^{T} \mathbf{Q g}-\mathbf{b}^{T} \mathbf{g}+c_{2}
$$

where $\mathbf{Q}$ was defined after (10), $\mathbf{b}$ $2 \operatorname{Re}\left(\int_{\mathcal{F}_{p}} e^{j \theta^{(d)}(f)} \sqrt{S(f)} Q^{*}(f) \mathbf{v}^{*}(f, L) d f\right), \quad$ and the constant $c_{2}=\int_{\mathcal{F}_{p}} S(f) d f$ is not required in the design problem. If we choose a matrix $\mathbf{L}$ such that $\mathbf{L}^{H} \mathbf{L}=\mathbf{Q}$, then when $\ell=2$, Problem 3 can be explicitly formulated as

$$
\begin{array}{ll}
\min _{\mathbf{g}, \mu} & \mu-\mathbf{b}^{T} \mathbf{g} \\
\text { s.t. } & \|\mathbf{L g}\|_{2}^{2} \leq \mu \\
& \|\mathbf{A}(f) \mathbf{g}\|_{2} \leq \sqrt{M(f)} \quad \text { for all } f \in\left[0, \frac{1}{\left(2 T_{0}\right)}\right] .
\end{array}
$$

This is a convex optimization problem with a linear objective, a rotated second-order cone constraint (25b), and an infinite number of convex quadratic constraints (25c). Discretization of (25c) results in a formulation that can be efficiently solved for a (globally) optimal g using general purpose convex cone optimization tools; e.g., [22]. Although solutions to (25) do not generate waveforms that explicitly maximize the NESP, they efficiently generate pulses with large NESPs, without the need for the spectral factorization post-processing step that is required for the efficient solution of Problem 1 [via (16)].

\section{Constrained Frequency Response Approximation With Linear Phase Filters}

The solution of Problem 3 can often be simplified if $g[k]$ is (further) constrained to have linear phase. While this additional constraint usually incurs a performance penalty, it leads to filters that have reduced storage requirements for their implementation. For brevity, we will focus on the case where the filter length is odd, $L=2 \tilde{L}+1$ and its impulse response is symmetric. In that case

$$
\begin{aligned}
G\left(e^{j 2 \pi f T_{0}}\right) & =e^{-j 2 \pi f T_{0} \tilde{L}} \sum_{k=\tilde{L}}^{L-1} g[k] e^{-j 2 \pi f T_{0}(k-\tilde{L})} \\
& =e^{-j 2 \pi f T_{0} \tilde{L}} \tilde{\mathbf{v}}^{T}(f, \tilde{L}+1) \tilde{\mathbf{g}}
\end{aligned}
$$

where $\tilde{\mathrm{g}}=[g[\tilde{L}], g[\tilde{L}+1], \ldots, g[L-1]]^{T}$. For such filters, the problem in (25) simplifies to

$$
\min _{\tilde{\mathbf{g}}, \mu} \mu-\tilde{\mathbf{b}}^{T} \tilde{\mathbf{g}}
$$

subject to

$$
\begin{aligned}
& \|\tilde{\mathbf{L}} \tilde{\mathbf{g}}\|_{2}^{2} \leq \mu \\
& -\sqrt{M(f)} \leq \tilde{\mathbf{v}}(f, \tilde{L}+1) \tilde{\mathbf{g}} \leq \sqrt{M(f)} \\
& \quad \text { for all } f \in\left[0, \frac{1}{\left(2 T_{0}\right)}\right]
\end{aligned}
$$

where $\tilde{\mathbf{Q}}=\tilde{\mathbf{L}}^{H} \tilde{\mathbf{L}}=\int_{\mathcal{F}_{p}} S_{q}(f) \tilde{\mathbf{v}}(f, \tilde{L}+1) \tilde{\mathbf{v}}^{T}(f, \tilde{L}+1) d f$ and $\tilde{\mathbf{b}}=2 \int_{\mathcal{F}_{p}} \cos \left(\theta^{(d)}(f)+2 \pi f T_{0} \tilde{L}-\theta_{q}(f)\right) \sqrt{S(f) S_{q}(f)}$ $\tilde{\mathbf{v}}(f, \tilde{L}+1) d f$. It is clear from the expression for $\tilde{\mathbf{b}}$ that a natural choice for $\theta^{(d)}(f)$ in a such a design is $\theta^{(d)}(f)=\theta_{q}(f)-2 \pi f T_{0} \tilde{L}$. We also point out that $(27 \mathrm{c})$ is a set of linear constraints (two for each $f$ ), whereas (25c) is a set of convex quadratic constraints. Once again, (27) can be efficiently solved using general purpose convex optimization tools, e.g., [22]. The problem in (27) is also particularly amenable to solution methods that employ multiple exchange techniques; e.g., [3] and [21].

The case of $\ell=\infty$ is also of interest because of its relationship with the PM designs. If we choose $\theta^{(d)}(f)=\theta_{q}(f)-$ $2 \pi f T_{0} \tilde{L}$, then for linear phase filters with a positive gain in the passband, we have the formulation (in epigraph form)

$$
\begin{array}{ll}
\min _{\tilde{\mathbf{g}}, t} & t \\
\text { s.t. } & \sqrt{\breve{M}(f)}-\frac{t}{\sqrt{S_{q}(f)}} \leq \tilde{\mathbf{v}}^{T}(f, \tilde{L}+1) \tilde{\mathbf{g}} \leq \sqrt{M(f)} \\
& \quad \text { for all } f \in \mathcal{F}_{p} \\
& t \leq \sqrt{S(f)}+\sqrt{M(f) S_{q}(f)} \quad \text { for all } f \in \mathcal{F}_{p} \\
& -\sqrt{M(f)} \leq \tilde{\mathbf{v}}^{T}(f, \tilde{L}+1) \tilde{\mathbf{g}} \leq \sqrt{M(f)} \quad \text { for all } f \notin \mathcal{F}_{p}
\end{array}
$$

where we have used the fact that (28b) implicitly constrains $t$ to be positive, and the fact that $\sqrt{M(f)} \leq \sqrt{\breve{M}(f)}+t / \sqrt{S_{q}(f)}$ for all $f \in\left[0,1 /\left(2 T_{0}\right)\right]$. The constraint in (28c) is required to ensure that the spectral mask is satisfied throughout the passband. The problem in (28) is an SILP in $\mathrm{g}$ and $t$, and hence it can be efficiently solved.

The formulation in (28) solves the following problem for an odd-length symmetric filter:

$$
\begin{aligned}
& \min _{\tilde{\mathbf{g}}} \max _{f \in \mathcal{F}_{p}} \sqrt{S_{q}(f)}\left|\sqrt{\breve{M}(f)}-\tilde{G}\left(e^{j 2 \pi f T_{0}}\right)\right| \\
& \text { subject to }\left|\tilde{G}\left(e^{j 2 \pi f T_{0}}\right)\right| \leq \sqrt{M(f)}
\end{aligned}
$$

where $\tilde{G}\left(e^{j 2 \pi f T_{0}}\right)=\tilde{\mathbf{v}}^{T}(f, \tilde{L}+1) \tilde{\mathbf{g}}$ is the "phase centered" version of $G\left(e^{j 2 \pi f T_{0}}\right)$. It is interesting to compare this problem to that solved in a previous approach to DSP-based UWB pulse design [13]. The design problem considered in [13] can be paraphrased as: given a set of frequency bands $\left\{\mathcal{F}_{s_{i}}\right\}_{i=1}^{N_{s}}$, known as stopbands, which are separated from $\mathcal{F}_{p}$ by so-called transition bands, find a linear phase filter which achieves

$$
\begin{aligned}
\min _{\tilde{\mathrm{g}}} \max \left\{\max _{f \in \mathcal{F}_{p}}\left|\sqrt{\breve{M}(f)}-\delta-\tilde{G}\left(e^{j 2 \pi f T_{0}}\right)\right|,\right. \\
\left.\eta_{i} \max _{f \in \mathcal{F}_{s_{i}}}\left|\tilde{G}\left(e^{j 2 \pi f T_{0}}\right)\right|\right\}_{i=1}^{N_{s}}
\end{aligned}
$$

where $\delta$ is a small positive constant to account for "ripples" in the passband and $\left\{\eta_{i}\right\}$ is a set of weights. This problem is directly solvable using the conventional Parks-McClellan algorithm [14]. While (29) emphasizes the fact that the waveform generated by the solution to (28) fully complies with the spectral mask, (30) illustrates that the equiripple nature of the filters designed by the PM algorithm may result in the mask being violated. In order to ensure mask compatibility of waveforms designed via the PM algorithm, the stopbands $\left\{\mathcal{F}_{s_{i}}\right\}$, the ripple tolerance $\delta$, and the weights $\left\{\eta_{i}\right\}$ must be carefully selected. The 


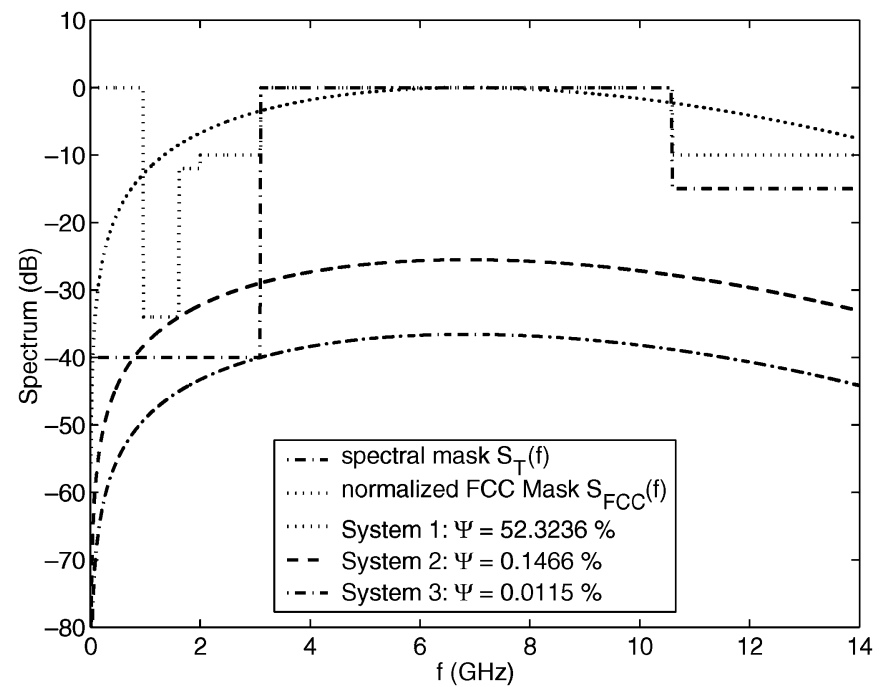

Fig. 2. Power spectrum of the Gaussian monocycle; System 1: complying to FCC mask only at UWB passband. System 2: strictly complying to FCC mask at all bands. System 3: strictly complying to the stricter mask at all bands.

search for appropriate values for these parameters would typically involve repeatedly solving (30) with different parameter values in an interactive fashion, until an acceptable waveform is found. In contrast, each of our proposed designs requires the solution of only one optimization problem, and that problem can be efficiently solved for a globally optimal waveform.

\section{E. Imperfect Knowledge of $Q(f)$}

The formulations of Problems 1 and 2 were based on precise knowledge of $S_{q}(f)$. In the case where $S_{q}(f)$ is not precisely known, we can replace $S_{q}(f)$ by an estimate $\hat{S}_{q}(f)$ that is also an upper bound; i.e., $\hat{S}_{q}(f) \geq S_{q}(f)$ for all $f$. This replacement is well motivated for design convenience, because $S_{q}(f)$ is typically fairly flat over the band of interest and because replacing $S_{q}(f)$ by a constant $\hat{S}_{q} \geq S_{q}(f)$, for all $f \in \mathcal{F}_{p}$, enables the integrals which constitute $\mathbf{Q}, \mathbf{c}$, and $\mathbf{b}$ to be analytically evaluated. Also, if $\hat{S}_{q}(f)$ is constant for all $f \in \mathcal{F}_{p}$, the precise transformation of some semi-infinite linear constraints into (finite) linear matrix inequalities [10] takes a relatively simple form. The natural formulations of Problem 3 depend on $Q(f)=$ $e^{j \theta_{q}(f)} \sqrt{S_{q}(f)}$, but as we demonstrated in our development, judicious choice of $\theta^{(d)}(f)$ results in a problem that depends only on $S_{q}(f)$, and hence similar approximations can be applied when $S_{q}(f)$ is not precisely known.

\section{F. Design Examples}

We now apply our formulations to the design of UWB pulses which satisfy the US FCC mask. We choose the basis pulse to be the Gaussian monocycle $q(t)=2 A \sqrt{e}(t / \tau) e^{-2(t / \tau)^{2}}$ [25], with its peak frequency response located at $1 /(4 \tau)=6.85 \mathrm{GHz}$. The clock rate $F_{0}$ is set to be a relatively low frequency of $1 / T_{0}=28 \mathrm{GHz}$. Based on the FCC spectral mask $S_{\mathrm{FCC}}(f)$ illustrated in Fig. 2, we observe that most of the UWB signal power should be allocated to the band from 3.1 to $10.6 \mathrm{GHz}$, while considerable attenuation is imposed in other regions of the spectrum, especially for frequencies up to $3.1 \mathrm{GHz}$. These constraints are designed to avoid interference to legacy narrow-band systems such as the Global Positioning System that resides in the frequency band $[0.96,1.61] \mathrm{GHz}$. Accordingly, we define $\mathcal{F}_{p}:=\{f \mid f \in[3.1,10.6] \mathrm{GHz}\}$ as the UWB passband. In addition to designs that comply with the FCC mask $S_{\mathrm{FCC}}(f)$, we also seek pulse designs that comply with a tighter mask $S_{\mathrm{T}}(f) \leq S_{\mathrm{FCC}}(f)$, given by (see Fig. 2)

$$
S_{\mathrm{T}}(f)=\left\{\begin{array}{ll}
0 \mathrm{~dB} & 3.1 \mathrm{GHz} \leq f \leq 10.6 \mathrm{GHz} \\
-40 \mathrm{~dB} & 0 \leq f<3.1 \mathrm{GHz} \\
-15 \mathrm{~dB} & f>10.6 \mathrm{GHz}
\end{array} .\right.
$$

Enforcing the tighter mask allows some margin for "spectral regrowth" due to nonlinearities in the transmitter.

To motivate the need for our design techniques, we first consider how well the standard Gaussian monocycle is matched to the spectral mask. For a Gaussian monocycle, we can scale the amplitude $A$ to generate three system designs, as illustrated in Fig. 2. System 1 complies with the FCC mask only in the UWB passband, System 2 has the largest possible amplitude under the FCC mask, and System 3 complies with the tighter mask $S_{\mathrm{T}}(f)$. Clearly, System 1 violates the FCC mask in the stopbands, whereas Systems 2 and 3 comply with their respective spectral masks at the expense of very low spectrum utilization.

1) Spectral Utilization Problem: We now investigate the power utilization efficiency of the proposed pulse designs.

- Design 1: Our first design is obtained by solving a version of (16) in which (16b) is discretized and (16c) is precisely transformed into $L$ linear constraints on an $L \times L$ positive semidefinite matrix (e.g., [10]). This transformation leads to an SDP formulation which can be efficiently solved for the optimal $\tilde{\mathbf{r}}$ using a general purpose solver; e.g., [22]. Furthermore, there are some indications (e.g., [4]) that the inherent structure in SDP can be exploited using specially designed algorithms that are substantially more efficient than general purpose methods. Spectral factorization [12], [26] is then applied to extract the optimal coefficients $g$.

- Design 2: In this case we design an odd-length, symmetric, linear phase filter via (27) in which (27c) is discretized. The resulting optimization problem has a linear objective, a set of linear constraints, and a single rotated second-order cone constraint.

We now compare the pulses generated by the above two design methods with those generated by several existing methods, including the PM pulse shaper in [13], the "prolate-spheroidal" (PS) pulse shaper in [17], and the Gaussian monocycle. All the pulses are configured to operate at the highest spectrum utilization while conforming to the relevant spectral mask. We will take the PM-based design for the FCC spectral mask in Section VI-A as the benchmark design. That design has a length $L=33$ filter, and the spectrum of the synthesized pulse is provided in Fig. 3. The power spectrum of a length $L=33$ PS pulse [17] is shown in Fig. 4. For the Gaussian monocycle, we employ Systems 2 and 3 in Fig. 2, as they do not violate their respective masks. The power spectra of the pulses emanating from Proposed Designs 1 and 2 for length $L=33$ filters and the FCC mask are provided in Fig. 5, from which the improved NESP of the proposed designs is immediately apparent. (The power spectrum of a pulse obtained from Proposed Design 1 with the tighter mask $S_{\mathrm{T}}(f)$ and a different basis pulse appeared in [27].) In order to quantify the improved NESPs of our designs, we have tabulated the NESPs of the different design methods under each spectral mask in Table I. 


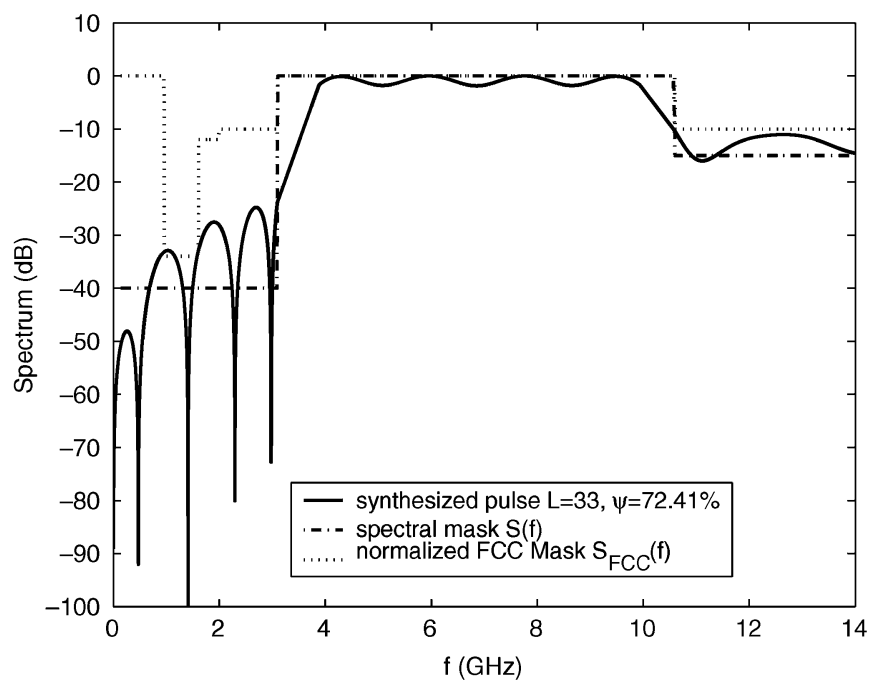

Fig. 3. Power spectrum of the PM algorithm based pulse [13] under the FCC mask $\mathrm{S}_{\mathrm{FCC}}(f)$.



Fig. 4. Power spectrum of the "prolate-spheroidal" pulse [17].

TABLE I

NESPS OF VARIOUS WAVEFORM DESIGNS

\begin{tabular}{l|l|l}
\hline Design Method & $S(f)=S_{\mathrm{T}}(f)$ & $S(f)=S_{\mathrm{FCC}}(f)$ \\
\hline Proposed Design 1: & $\bar{\psi}=82.08 \%$ & $\bar{\psi}=92.16 \%$ \\
Proposed Design 2: & $\bar{\psi}=76.51 \%$ & $\bar{\psi}=88.98 \%$ \\
PM Pulse [13]: & $\bar{\psi}=70.48 \%$ & $\bar{\psi}=72.41 \%$ \\
PS Pulse [17]: & $\bar{\psi}=32.11 \%$ & $\bar{\psi}=32.11 \%$ \\
Gaussian Monocycle: & $\bar{\psi}=0.0115 \%$ & $\bar{\psi}=0.15 \%$ \\
\hline
\end{tabular}

As expected, Proposed Design 1 provides the largest NESP, because it is based on direct maximization of the NESP (see Problem 1). However, it is interesting to note that both this

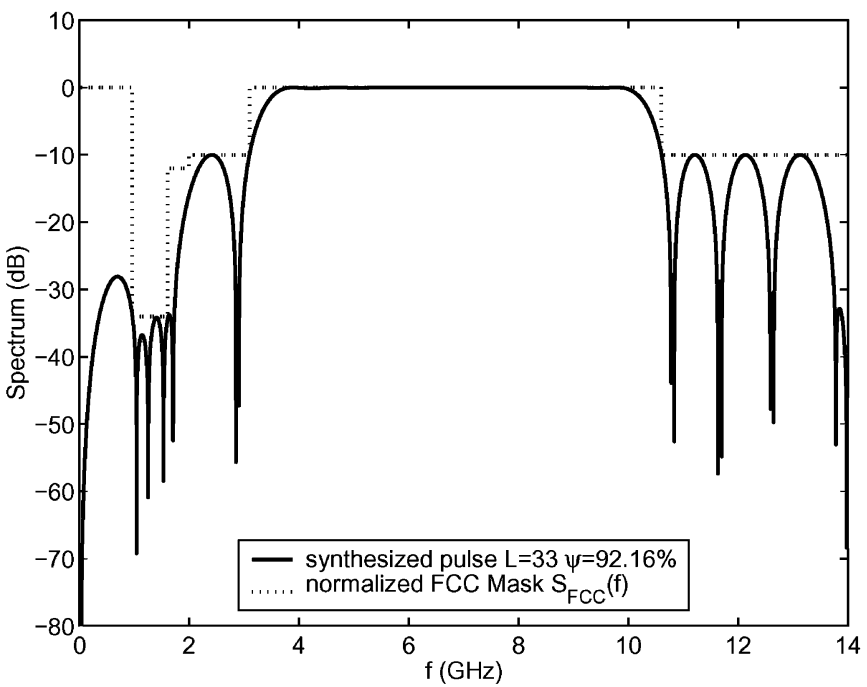

(a)

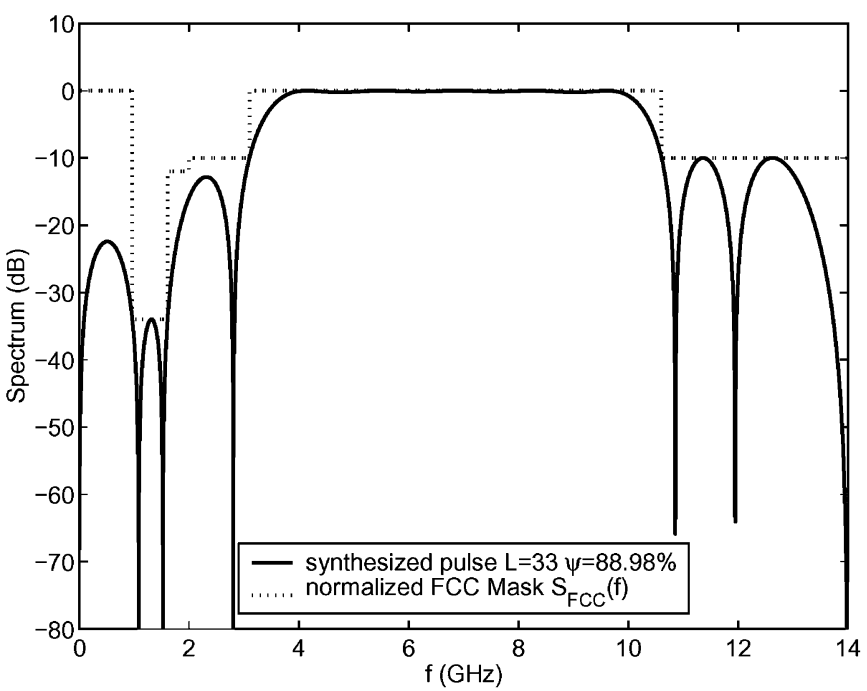

(b)

Fig. 5. Power spectrum of the optimally synthesized pulse under the FCC mask $\mathrm{S}_{\mathrm{FCC}}(f)$ : (a) Design 1 and (b) Design 2.

design and Proposed Design 2 (which is based on the indirect method of Problem 3 with the linear phase constraint) provide considerably larger NESPs than the PM pulse and substantially larger NESPs than the PS design and the Gaussian monocycle. This performance advantage is more pronounced when the FCC mask is selected rather than the tighter mask $S_{\mathrm{T}}(f)$. These comparisons demonstrate the flexibility of our design approach in accommodating different pulse design requirements while achieving the largest possible NESPs. The proposed designs (and the PM-based pulses) also enjoy an implementation advantage in terms of their low sampling frequency requirement compared to that of the PS pulses $(64 \mathrm{GHz})$ [17].

2) Pulse Duration Problem: Instead of maximizing the NESP over filters of a given length, an alternative design problem is to find the shortest pulse that achieves a given NESP requirement, subject to satisfaction of the spectral mask. Since the pulse duration is essentially determined by the filter length $L$, we can cast this design problem as: Given $T_{0}, S(f)$, $S_{q}(f)$ and $\bar{\gamma}$, find a filter $\mathrm{g}$ that has the minimum length $L$, 


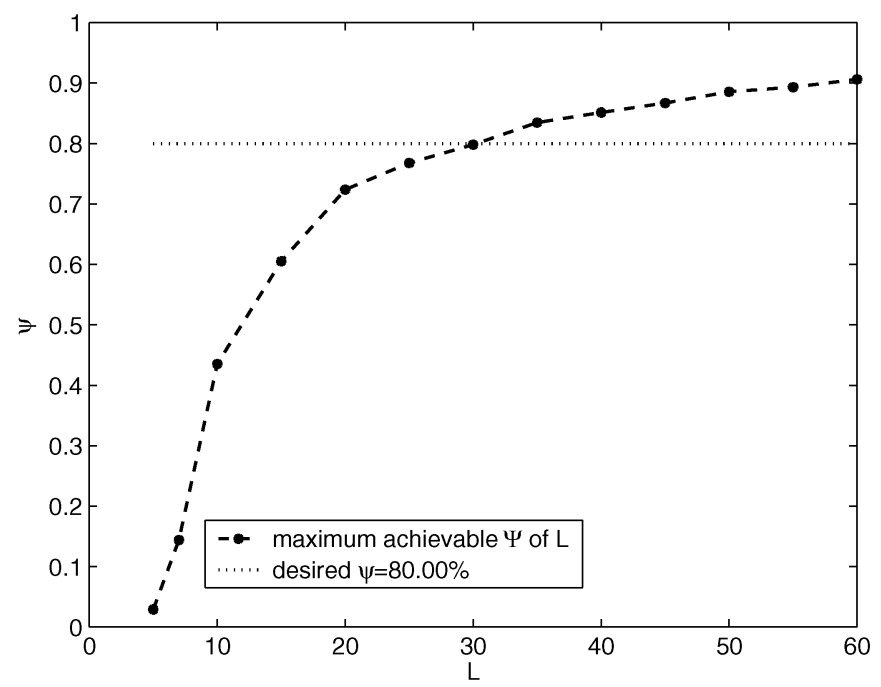

Fig. 6. Maximum achievable NESP $\psi$ versus $L$ under the tighter mask $\mathrm{S}_{r m T}(f)$.

subject to $\bar{\psi} \geq \bar{\gamma}$ and $S_{p}(f) \leq S(f)$ for all $f$. By defining $\gamma=\bar{\gamma} \int_{\mathcal{F}_{p}} S(\bar{f}) d f$, this problem can be formulated in terms of the autocorrelation $\tilde{\mathbf{r}}$ as follows:

$$
\begin{array}{ll}
\min _{\tilde{\mathbf{r}}, L} & L \\
\text { s.t. } & \mathbf{c}^{T} \tilde{\mathbf{r}} \geq \gamma \\
& \tilde{\mathbf{v}}^{T}(f, L) \tilde{\mathbf{r}} \leq M(f) \quad \text { for all } f \in\left[0, \frac{1}{\left(2 T_{0}\right)}\right] \\
& \tilde{\mathbf{v}}^{T}(f, L) \tilde{\mathbf{r}} \geq 0 \quad \text { for all } f \in\left[0, \frac{1}{\left(2 T_{0}\right)}\right] .
\end{array}
$$

This problem is quasi-convex (in the sense of [6, Section 4.2.5]), and hence it can be solved by a bisection search on $L$ for the feasibility/infeasibility boundary of (32). [The smallest $L$ for which (32b)-(32d) are simultaneously satisfiable is the solution.] At each stage of this search we solve efficiently the semi-infinite linear feasibility problem in (32) for the current value of $L$.

To demonstrate the application of this approach, we determined the shortest filter that can generate a pulse with an NESP of at least $80 \%$ under the (tighter) mask constraint $S_{\mathrm{T}}(f)$. (Recall that when $L=33$, the maximum achievable NESP is $82.08 \%$.) After the bisection search, we found that when $L=$ 31 , one has $\psi=81.25 \%$; and when $L=30, \psi=79.81 \%$. Choosing $L=31$, we obtain our optimally synthesized pulse $p(t)$ of duration $T_{p}=1.65 \mathrm{~ns}$. Similarly, the minimum length filter required to achieve the NESP of $72.41 \%$ that was achieved by the length 33 PM filter is mere $L=21$. In order to gain further insight, in Fig. 6 we have plotted the tradeoff between the filter length and the NESP for the tighter mask $S_{\mathrm{T}}(f)$. No point above the curve is achievable, and filters which achieve points on the curve can be efficiently obtained by solving Problem 1 via (16). Fig. 6 is instrumental in striking a balance between performance (spectral utilization efficiency $\bar{\psi}$ ) and complexity (filter length $L$ ). The NESP $\bar{\psi}$ increases rapidly for small to medium lengths but increases fairly slowly for longer filters.

3) Extension to Multiband Pulse Design: An alternative signaling format to single-band UWB impulse radio is to use multiband (MB) UWB waveforms, which have been proposed for wireless personal area networks (WPANs) under IEEE 802.15
[5]. In a multiband system, multiple bands of bandwidth greater than or equal to $500 \mathrm{MHz}$ are employed, with each band being occupied by a distinct pulse. With the entire bandwidth divided into several nonoverlapping subbands, multiband UWB systems allow flexibility in efficiently "filling up" the spectral mask, and facilitate coexistence with legacy systems and worldwide deployment by enabling some subbands to be turned off in order to avoid interference and comply with different regulatory requirements. In addition, multiband systems provide another dimension for multiple access via frequency division. Different users can use different pulses for multiple access, and frequency hopping can also be easily implemented by switching among those baseband pulses to acquire greater frequency diversity.

Fortunately, pulse design for an MB system can directly borrow the results from single-pulse design. The only difference lies in the different specifications of passband and the corresponding spectral mask constraint. We assume that the passband is equally divided into $K$ subbands, each of bandwidth of $B_{s}=(7.5 / K) \mathrm{GHz}$. For the pulse that occupies the $k$ th subband, $1 \leq k \leq K$, we impose a spectral mask $S_{k}(f)$ that satisfies the global spectral mask and has low stopband levels in order to avoid significant interband interference. In particular, we impose

$$
S_{k}(f)= \begin{cases}-60 \mathrm{~dB} & {\left[0,3.1+(k-1) B_{s}\right] \mathrm{GHz}} \\ 0 \mathrm{~dB} & {\left[3.1+(k-1) B_{s}, 3.1+k B_{s}\right] \mathrm{GHz} .} \\ -60 \mathrm{~dB} & {\left[3.1+k B_{s},+\infty\right) \mathrm{GHz}}\end{cases}
$$

The filter tap coefficients $\mathrm{g}_{k}$ for the $k$ th pulse can be obtained by efficiently solving Problem 1 via the formulation in (16). As an example, we suppose that the UWB passband is equally divided into three subbands. Setting $L=100$, we obtain the synthesized pulses $\left\{p_{k}(t)\right\}_{k=1}^{3}$ by replacing $S(f)$ in Problem 1 with $S_{k}(f)$ in (33), $k=1,2,3$, respectively. The power spectra of the synthesized multiband pulses are shown in Fig. 7.

\section{Optimal UWB ORThOgOnal Pulse Design}

Although they are (almost) orthogonal to each other, each of the pulses in a multiband UWB system can only utilize a portion of the power allowed by the FCC mask, since each of them occupies only a portion of the entire bandwidth. The need for higher data rates requires higher transmission power, which, in turn, calls for orthogonal pulse sets with better spectrum utilization. In addition to offering enhanced multipath diversity, orthogonal pulses with overlapping spectra stand out as a promising alternative to fulfill the need for both orthogonal multiple access and high data rates. In this section, we extend our FIR prefiltering structure to deal with the orthogonal pulse design problem.

\section{A. Orthogonality Formulation}

Let us consider two pulses $p_{1}(t)$ and $p_{2}(t)$ that are generated by two different sets of filter coefficients $\mathbf{g}_{1}$ and $\mathbf{g}_{2}$, each of length $L$. For these two pulses to be orthogonal, they have to satisfy the time-domain constraint $\int_{-\infty}^{+\infty} p_{1}(t) p_{2}(t) d t=0$, which can be equivalently written in the frequency domain as $\int_{-\infty}^{+\infty} P_{1}(f) P_{2}^{*}(f) d f=0$. In a matrix-vector form, the orthogonality constraint can be written as

$$
\int_{-\infty}^{+\infty} P_{1}(f) P_{2}^{*}(f) d f=\mathrm{g}_{1}^{H} \mathbf{Q g}_{2}=0
$$




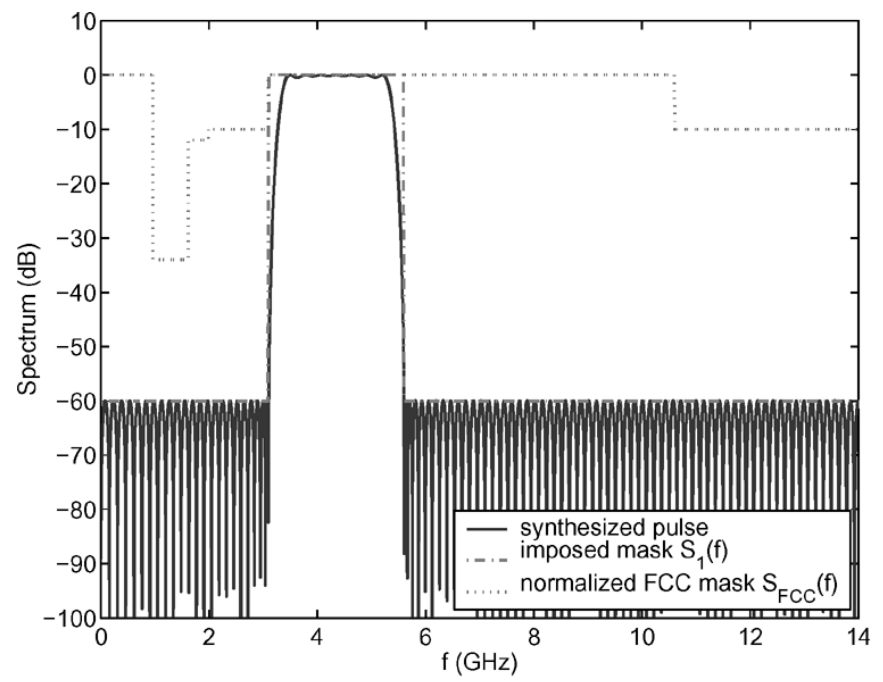

(a)

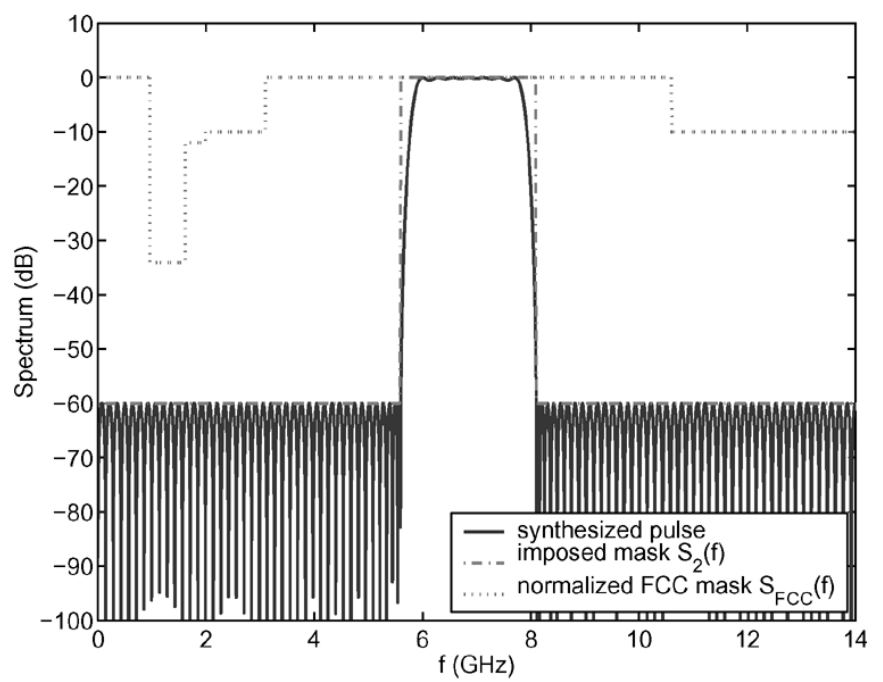

(b)

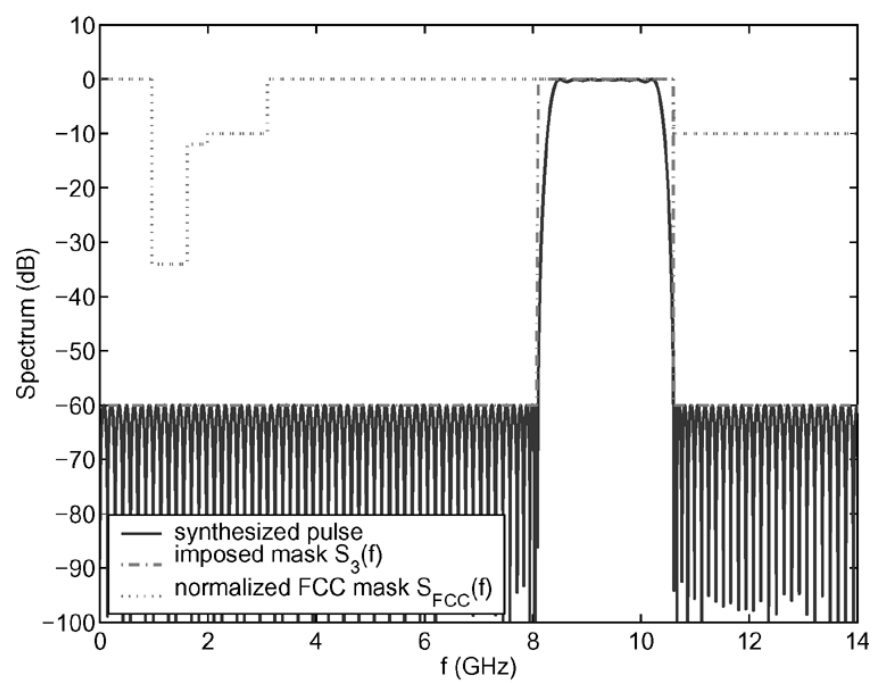

(c)

Fig. 7. Results for a three-band multiband UWB system. (a) First subband; (b) second subband; (c) third subband.

where $\mathbf{Q}$ was defined after (10). Suppose that a filter $\mathbf{g}_{1}$ has been designed via a single pulse method, such as Problem 1. The problem of directly maximizing the NESP of $p_{2}(t)$ subject to the orthogonality constraint (34) can be formulated as follow.

Orthogonal Pulse Design 1: Given $L, T_{0}, S_{q}(f), M(f)$, and $\mathrm{g}_{1}$, find $\mathrm{g}_{2}$ that achieves

$$
\begin{aligned}
& \underset{\mathbf{g}_{2}}{\max } \quad \psi=\mathbf{g}_{2}^{T} \mathbf{Q} \mathbf{g}_{2} \\
& \text { such that } \quad \mathbf{g}_{1}^{T} \mathbf{Q} \mathbf{g}_{2}=0 \\
& \qquad \mathbf{A}(f) \mathbf{g}_{2} \|_{2} \leq \sqrt{M(f)} \forall f \in\left[0, \frac{1}{\left(2 T_{0}\right)}\right]
\end{aligned}
$$

or show that none exists.

The linear equality constraint in (35b) and the set of convex quadratic constraints in (35c) describe a convex feasible set, but, as in (14), the objective is to maximize a convex function of $\mathrm{g}_{2}$, and hence (35) is a nonconvex optimization problem. Unfortunately, it is not possible to transform (35b) into a function of the autocorrelation vector $\tilde{\mathbf{r}}_{2}$, and therefore we cannot directly borrow the direct single pulse design techniques from Section III-A, as we did for the multiband designs. In order to avoid the intricacies of having to deal with the potential for locally optimal solutions in the solutions of (35), we now seek formulations of an indirect design problem that is easier to solve. Our indirect formulations are based on the frequency response approximation problem discussed in Section III-C.

As shown in Section III-C, a pulse with a large NESP can be efficiently obtained by making $P_{k}(f)$ close to a desired frequency response $P_{k}^{(d)}(f):=e^{j \theta_{k}^{(d)}(f)} \sqrt{S(f)}$. When designing multiple orthogonal pulses, the desired response is constructed so that each $P_{k}^{(d)}(f)$ has the same power spectrum $S(f)$ but a distinct phase $\theta_{k}^{(d)}(f)$. To impose orthogonality among different pulses, we can select the design parameters $\left\{\theta_{k}^{(d)}(f)\right\}$ such that the desired $\left\{P_{k}^{(d)}(f)\right\}$ are mutually orthogonal. That is

$$
\int e^{j\left(\theta_{k}^{(d)}(f)-\theta_{i}^{(d)}(f)\right)} S(f) d f=0 \quad \text { for any } k \neq i .
$$

If the desired frequency responses are orthogonal, then the designed pulses will (essentially) inherit this property if the achieved approximation error is sufficiently small..$^{5}$ We can formulate the resulting design problem as follows.

Orthogonal Pulse Design 2: Given $L, T_{0}, \theta_{q}(f), M(f)$, a filter $\mathbf{g}_{1}$ designed via (23), and $\theta_{2}^{(d)}(f)$ that is orthogonal to $\theta_{1}^{(d)}(f)$ according to (36), find $\mathbf{g}_{2}$ that achieves

$$
\min _{\mathbf{g}_{2}} \quad \phi_{\ell}=\left\|e^{j \theta_{2}^{(d)}(f)} \sqrt{S(f)}-Q(f) G_{2}\left(e^{j 2 \pi f T_{0}}\right)\right\|_{\mathcal{L}_{\ell}\left(\mathcal{F}_{p}\right)}
$$

$$
\text { such that } \quad\left\|\mathbf{A}(f) \mathbf{g}_{2}\right\|_{2} \leq \sqrt{M(f)} \quad \forall f \in\left[0, \frac{1}{\left(2 T_{0}\right)}\right]
$$

or show that none exists.

An approximation of the orthogonality constraint in (36) can significantly simplify the design of (essentially) orthogonal pulses. In particular, since $\mathcal{F}_{p}$ is the passband (or bands) of the spectral mask, then the constraint in (36) can be approximated by taking the integral over $\mathcal{F}_{p} \cup \tilde{\mathcal{F}}_{p}$, where $\tilde{\mathcal{F}}_{p}$ is the mirror

${ }^{5}$ During the pulse shape design phase, if the channel information is available at the transmitter, it can be incorporated into (36). By doing so, we can ensure orthogonality among pulses even at the receiver's end. 
image of $\mathcal{F}_{p}$ in the negative frequencies. If $S(f)$ is constant over $\mathcal{F}_{p}$, then (36) can be approximated by

$$
\int_{\mathcal{F}_{p} \bigcup \tilde{\mathcal{F}}_{p}} e^{j\left(\theta_{k}^{(d)}(f)-\theta_{i}^{(d)}(f)\right)} d f=0 \text { for any } k \neq i .
$$

If that integral is (further) approximated by its $N$-point centred Riemann sum and $N$ is large, then the desired responses will be (essentially) orthogonal if

$$
\mathbf{w}_{i}^{H} \mathbf{w}_{k}+\mathbf{w}_{k}^{H} \mathbf{w}_{i}=0 \quad \text { for any } k \neq i
$$

where the $n$th element of the phase vector $\mathbf{w}_{k}$ is $\mathbf{w}_{k}[n]=$ $e^{j \theta_{k}^{(d)}\left(f_{n}\right)}, 0 \leq n \leq N-1$, with $f_{n}=f_{\text {left }}+(n+1 / 2) \Delta_{f}, f_{\text {left }}$ being the lower band edge of $\mathcal{F}_{p}$ and $\Delta_{f}$ being the width of the Riemann rectangle, which is $(1 / N)$ th of the bandwidth of $\mathcal{F}_{p}$. For convenience, we will define $\mathrm{F}_{p}^{N}$ to be the set of such $f_{n}$ 's. A sufficient condition for (39) is that $\mathbf{w}_{k}^{H} \mathbf{w}_{i}=0$ for any $k \neq i$.

\section{B. Sequential UWB Pulse Design}

Having understood the orthogonality requirements, we now propose a sequential (SEQ) strategy for (essentially) orthogonal pulse design. The procedure starts with the design of the first pulse $p_{1}(t)$ subject to the mask constraint only. Subsequent pulses $p_{k}(t), k=2, \ldots, K$, are then designed one by one to fit into the desired spectral mask, as well as to be (essentially) orthogonal to all previously designed pulses. To describe mathematically, we suppose that $(k-1)$ (essentially) mutually orthogonal pulses $\left\{p_{m}(t)\right\}_{m=1}^{k-1}$ are already in place. Rather than directly minimizing the approximation error of the $k$ th pulse $\left\|P_{k}^{(d)}(f)-P_{k}(f)\right\|_{\mathcal{L}_{\ell}\left(\mathcal{F}_{p}\right)}$, we minimize the $\ell$-norm of an $N$-element vector $\mathbf{x}$ whose $n$th element is $P_{k}^{(d)}\left(f_{n}\right)-P_{k}\left(f_{n}\right), f_{n} \in \mathrm{F}_{p}^{N}$. When $\ell<\infty$, this corresponds to minimizing the $N$-point centred Riemann sum approximation of $\left\|P_{k}^{(d)}(f)-P_{k}(f)\right\|_{\mathcal{L}_{\ell}\left(\mathcal{F}_{p}\right)}$. Since $P_{k}^{(d)}\left(f_{n}\right)=\mathbf{w}_{k}[n] \sqrt{S\left(f_{n}\right)}$, the subproblem in the SEQ strategy can be formulated as follows.

SEQ Pulse Design: Given $\ell, L, T_{0}, S_{q}(f), S(f), N$, and $k-$ 1 previously selected filter phase vectors $\left\{\mathbf{w}_{m}\right\}_{m=1}^{k-1}$ of mutually orthogonal pulses $\left\{p_{m}(t)\right\}_{m=1}^{k-1}$, find vectors $\mathbf{g}_{k}$ of length $L$ and $\mathbf{w}_{k}$ of length $N$ that satisfy

$$
\begin{aligned}
& \min _{\mathbf{g}_{k}, \mathbf{w}_{k}, \mathbf{x}}\|\mathbf{x}\|_{\ell} \\
& \text { such that } \quad \mathbf{x}[n]=\mathbf{w}_{k}[n] \sqrt{S\left(f_{n}\right)}-Q\left(f_{n}\right) \mathbf{v}^{H}\left(f_{n}, L\right) \mathbf{g}_{k} \\
& \forall f_{n} \in \mathbf{F}_{p}^{N} \\
& \left\|\mathbf{A}(f) \mathbf{g}_{k}\right\|_{2} \leq \sqrt{M(f)}, \quad \forall f \in\left[0, \frac{1}{\left(2 T_{0}\right)}\right]
\end{aligned}
$$$$
\mathbf{w}_{k}^{H} \mathbf{w}_{m}=0, \quad \forall m=1, \ldots, k-1
$$

or show that none exists.

In this formulation, the objective (40a) [and (40b)] indirectly seeks a pulse with a large NESP, while (40c) and (40d) ensure spectral mask compatibility and mutual orthogonality of the target spectra, respectively.
To reduce the complexity of searching for $\mathbf{w}_{k}$, a convenient approach is to select mutually orthogonal phase vectors prior to the SEQ procedure. Treating $\mathbf{w}_{k}$ as a length- $N$ codeword with complex-valued elements, we can use the fast Fourier transform (FFT) matrix to design an example of $K$ codewords as follows.

Complex Orthogonal Phase Vectors via FFT: A set of $K$ mutually orthogonal phase vectors $\left\{\mathbf{w}_{k}\right\}_{k=1}^{K}$ can be obtained by setting $\mathbf{w}_{k}[n]=e^{j 2 \pi k n / N}, n=0, \ldots, N-1$, for $k=$ $1, \ldots, K \leq N$.

With the phase vectors $\left\{\mathbf{w}_{k}\right\}$ selected this way, we now mimic (25) to propose the following convex formulation of the SEQ pulse design subproblem with $\ell=2$ :

$$
\begin{aligned}
& \min _{\mathbf{g}_{k}, \mu_{k}} \quad \mu_{k}-\hat{\mathbf{b}}_{k}^{T} \mathbf{g}_{k} \\
& \text { such that } \quad\left\|\mathbf{L g}_{k}\right\|_{2}^{2} \leq \mu_{k}, \\
& \qquad\left\|\mathbf{A}(f) \mathbf{g}_{k}\right\|_{2} \leq \sqrt{M(f)} \forall f \in\left[0, \frac{1}{\left(2 T_{0}\right)}\right]
\end{aligned}
$$

where $\hat{\mathbf{b}}_{k}=2 \Delta_{f} \operatorname{Re}\left(\sum_{n=0}^{N-1} \mathbf{w}_{k}[n] \sqrt{S\left(f_{n}\right)} Q^{*}\left(f_{n}\right) \mathbf{v}^{*}\left(f_{n}, L\right)\right)$.

\section{Sequential UWB Pulse Design With Linear Phase Filters}

The formulation in (41) allows the FIR filters to have nonlinear phase characteristics. The design can be simplified if $g[k]$ is further constrained to have linear phase, which in turn simplifies the selection of the orthogonal phase vectors $\mathrm{w}_{k}$. In the case of odd-length symmetric linear phase filters, (40b) simplifies to

$$
\begin{aligned}
\mathbf{x}[n]= & \mathbf{w}_{k}[n] \sqrt{S\left(f_{n}\right)} \\
& -\sqrt{S_{q}\left(f_{n}\right)} e^{j\left(\theta_{q}\left(f_{n}\right)-2 \pi f_{n} T_{0} \tilde{L}\right)} \tilde{\mathbf{v}}^{T}\left(f_{n}, \tilde{L}+1\right) \tilde{\mathbf{g}} .
\end{aligned}
$$

Since $\tilde{\mathbf{v}}^{T}(f, \tilde{L}+1) \tilde{\mathbf{g}}$ is real, a natural choice for $\mathbf{w}_{k}[n]$ is $\mathbf{w}_{k}[n]=e^{j\left(\theta_{q}\left(f_{n}\right)-2 \pi f_{n} T_{0} \tilde{L}\right)} \tilde{\mathbf{w}}_{k}[n]$, where $\tilde{\mathbf{w}}_{k}[n]= \pm 1$. That is, the orthogonal codewords can be constructed from binary orthogonal codewords, $\tilde{\mathbf{w}}_{k}$. If we choose $N$ to be a power of two, with $N \geq K$, such binary codewords can be selected from the set of length $N=2^{i}$ Hadamard codewords; e.g., [18, p. 424]. When $K<2^{i}$, one must select elements from this set. In the Appendix, we argue that an appropriate method is to select Hadamard codewords in ascending order of the number of sign transitions in the codeword.

Binary Orthogonal Phase Vectors via Hadamard Partition: In a SEQ pulse design problem with linear phase filters, the binary codewords $\left\{\tilde{\mathbf{w}}_{k}\right\}$ of length $N=2^{i}$ should be selected from the set of length- $2^{i}$ Hadamard codewords as follows.

1) Arrange all Hadamard codewords in ascending order of the number of sign transitions in the codeword.

2) Set $\tilde{\mathbf{w}}_{k}$ to be the $k$ th Hadamard codeword.

This choice (essentially) minimizes the loss of NESP due to the "crossing bands" between bands of positive and negative gain in the passband of the filter. As an example, if we have $N=8$ discretization points (i.e., eight Riemann rectangles) and we want to design $K=3$ pulses, the Hadamard partition result suggests the following choices:

$$
\begin{aligned}
& \tilde{\mathbf{w}}_{1}=[1,1,1,1,1,1,1,1]^{T} \\
& \tilde{\mathbf{w}}_{2}=[1,1,1,1,-1,-1,-1,-1]^{T} \\
& \tilde{\mathbf{w}}_{3}=[1,1,-1,-1,-1,-1,1,1]^{T} .
\end{aligned}
$$




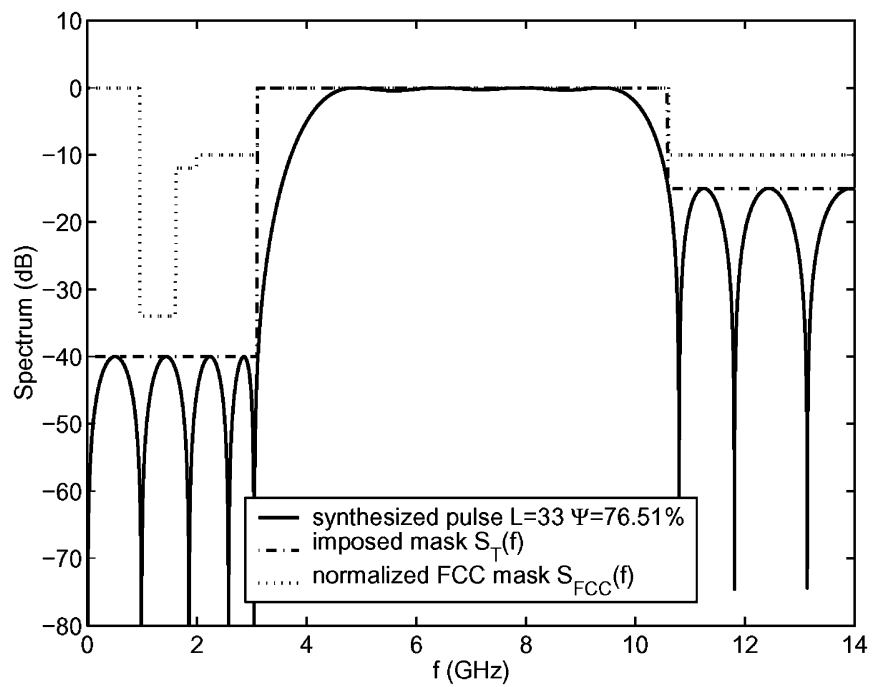

(a)

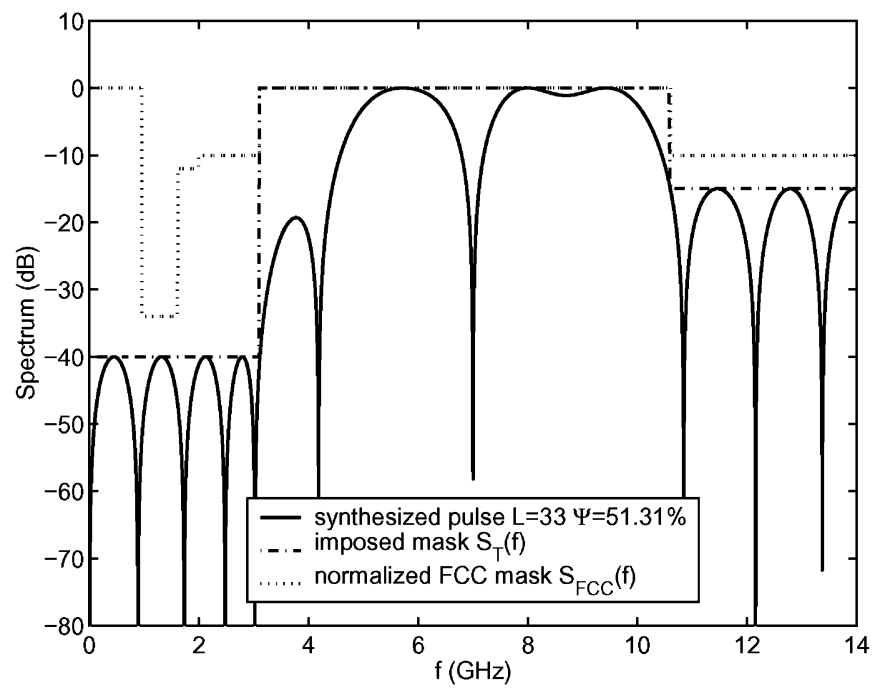

(b)

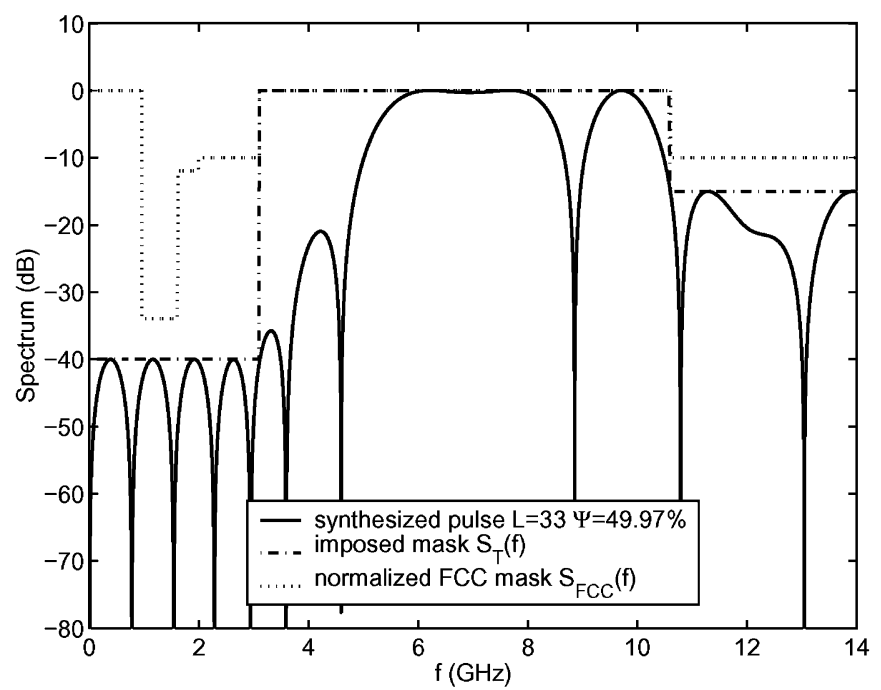

(c)

Fig. 8. Three orthogonal pulses designed via the SEQ strategy. (a) First pulse; (b) second pulse; (c) third pulse.

For the larger values of $N$ that would be used in practice, the first three codewords have a similar sign structure.

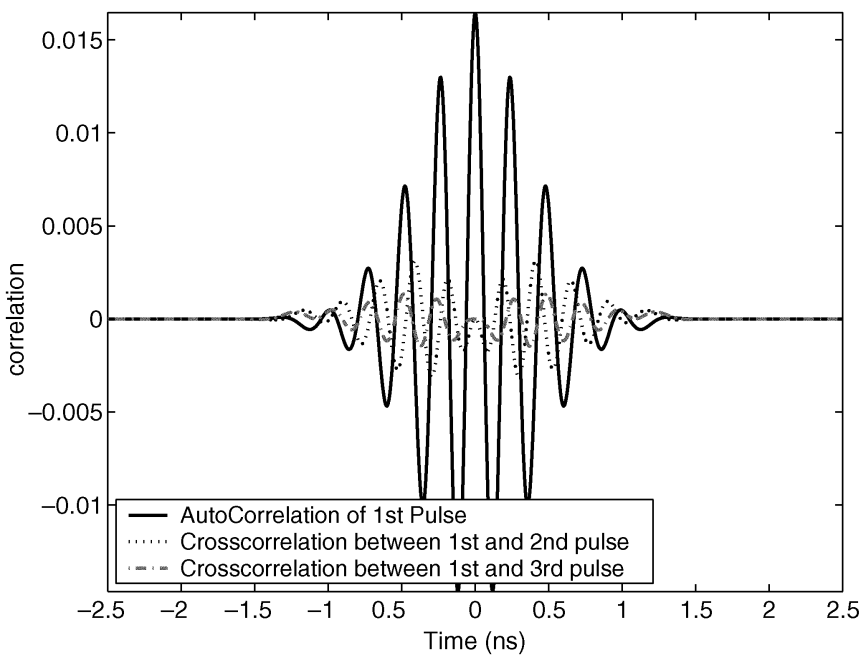

Fig. 9. Correlation properties of the designed orthogonal pulses.

With odd-length symmetric linear phase filters and the phase vectors chosen as described above, (40) simplifies to

$$
\begin{aligned}
\min _{\tilde{\mathbf{g}}_{k}, \mathbf{x}} & \|\mathbf{x}\|_{\ell} \\
\text { s.t. } \quad \mathbf{x}[n] & =\sqrt{S_{q}\left(f_{n}\right)} \\
\times & \left(\tilde{\mathbf{w}}_{k}[n] \sqrt{\breve{M}\left(f_{n}\right)}-\tilde{\mathbf{v}}^{T}\left(f_{n}, \tilde{L}+1\right) \tilde{\mathbf{g}}_{k}\right) \\
\forall f_{n} \in \mathrm{F}_{p}^{N} & \leq \tilde{\mathbf{v}}^{T}(f, \tilde{L}+1) \tilde{\mathbf{g}}_{k} \leq \sqrt{M(f)} \\
-\sqrt{M(f)} & \in\left[0, \frac{1}{\left(2 T_{0}\right)}\right]
\end{aligned}
$$

from which explicit formulations for $\ell=2$ and $\ell=\infty$ can be easily obtained.

\section{SEQ UWB Pulse Design Example}

As an example, we have designed three orthogonal UWB pulses using the sequential design strategy with linear phase filters described in the previous section, including the proposed selection of the Hadamard codewords. We chose length $L=33$ filters, length $N=512$ codewords, and the filters were designed using a formulation of (44) with $\ell=2$. Under the tighter mask constraint $S_{\mathrm{T}}(f)$, the power spectra of the resulting (essentially) orthogonal pulses are provided in Fig. 8. The correlation properties of these pulses with $p_{1}(t)$ are provided in Fig. 9. The NESPs of these three pulses are $76.51 \%, 51.31 \%$, and $49.97 \%$, respectively, and hence all three pulses provide higher spectral utilization efficiency than the pulse from the PS orthogonal set [17] with the largest spectral utilization efficiency. (The PS pulse has an NESP of $32.11 \%$, and its power spectrum is shown in Fig. 4.)

\section{CONCLUSION}

In this paper, we developed efficient methods for the design of FIR prefiltering structures that synthesize single pulses and sets of (essentially) orthogonal pulses for UWB systems that must satisfy a spectral mask constraint, such as that imposed by the U.S. FCC. Some algebraic transformations facilitated the 
formulation of various single pulse design problems as convex optimization problems, from which a globally optimal solution can be efficiently obtained. Related techniques were used to generate efficiently solvable formulations of a range of other UWB pulse design problems, including a minimum duration single-pulse design, multiband pulse design, and multiple orthogonal pulse design. For the orthogonal pulse design problem a sequential strategy was proposed, along with an optimal procedure for the selection of the phase of the desired responses. This approach was shown to generate a set of (essentially) orthogonal pulses with high spectral utilization. Compared to other existing pulses, our pulses provide better spectrum utilization and have a lower sampling frequency requirement.

\section{APPENDIX \\ NESP OF LINEAR PHASE FILTERS WITH HADAMARD DesiRed Phase Vectors}

In Section IV-C, we described a procedure for selecting the phase vectors $\mathbf{w}_{k}$ of the desired frequency response for linear phase filters that generate a set of (essentially) mutually orthogonal UWB pulses. To investigate how the proposed selection from the set of Hadamard codewords affects the achievable NESP for each pulse, we introduce the following definitions. First, we let $\delta$ be a small positive value which represents the magnitude of the allowable passband ripple of the pulse. For the $k$ th filter we have the following.

- A band of frequencies $f \in\left[f_{L}, f_{U}\right] \subseteq \mathcal{F}_{p}$ is said to constitute a positive passband if $\sqrt{\breve{M}(f)}-\delta \leq \tilde{\mathbf{v}}^{T}(f, \tilde{L}+$ 1) $\tilde{\mathbf{g}}_{k} \leq \sqrt{\breve{M}(f)}$ for all $f \in\left[f_{L}, f_{U}\right]$.

- That band is said to be a negative passband if $-\sqrt{\breve{M}(f)} \leq$ $\tilde{\mathbf{v}}^{T}(f, \tilde{L}+1) \tilde{\mathbf{g}}_{k} \leq-\sqrt{\breve{M}(f)}+\delta$ for all $f \in\left[f_{L}, f_{U}\right]$.

- Any band between a positive passband and a negative passband is said to be a crossing band.

- The frequencies at edges of $\mathcal{F}_{p}$ that are not in positive or negative passbands are said to be a transition band.

Given these definitions, it is clear from (44b) that the presence of a crossing band corresponds to a sign transition in $\tilde{\mathbf{w}}_{k}$.

Since $\left|P_{k}(f)\right|^{2}=S_{q}(f)\left|\tilde{\mathbf{v}}^{T}(f, \tilde{L}+1) \tilde{\mathbf{g}}_{k}\right|^{2}$, the total NESP of a waveform is the sum of the NESPs generated by the positive and negative passbands of the filter, plus that generated by the crossing and transition bands of the filter. Since the power spectrum of the pulse is close to the mask in the bands which correspond to the passbands of the filter, we have almost perfect spectral utilization in those bands. Therefore, the contributions of the passbands to the NESP is approximately $\left(1-\epsilon_{1}\right)\left(\beta_{+}+\beta_{-}\right)$, where $\epsilon_{1}$ is a small positive number, and $\beta_{ \pm}$is the fraction of the passband occupied by the positive (negative) passbands; i.e., $\beta_{ \pm}=B_{ \pm} / B$, where $B_{ \pm}$is the sum of the bandwidths of the positive/negative passbands and $B$ is the bandwidth of $\mathcal{F}_{p}$. In contrast, the power spectrum of the pulse is some distance from the mask in the bands corresponding to the crossing and transition bands of the filter, and hence the contribution of these bands to the NESP can be approximated by $\epsilon_{2}\left(\beta_{C}+\beta_{T}\right)$, where $\epsilon_{2}$ is a small number and $\beta_{C}$ and $\beta_{T}$ are the sums of the fractional bandwidths of the crossing and transition bands. Therefore, the overall NESP can be approximated by

$$
\begin{aligned}
\bar{\psi} & \approx\left(1-\epsilon_{1}\right)\left(\beta_{+}+\beta_{-}\right)+\epsilon_{2}\left(\beta_{C}+\beta_{T}\right) \\
& =\left(1-\epsilon_{1}\right)-\left(1-\epsilon_{1}-\epsilon_{2}\right)\left(\beta_{C}+\beta_{T}\right)
\end{aligned}
$$

where we have used the fact that $\beta_{+}+\beta_{-}+\beta_{C}+\beta_{T}=1$. Typically, the bands corresponding to the crossing bands will be about the same width, and hence $\beta_{C} \approx N_{\mathrm{cb}} \beta_{c, 1}$, where $N_{\mathrm{cb}}$ is the number of crossing bands and $\beta_{c, 1}$ is the fractional bandwidth of one crossing band. Hence

$$
\bar{\psi} \approx\left(1-\epsilon_{1}\right)-\left(1-\epsilon_{1}-\epsilon_{2}\right)\left(N_{\mathrm{cb}} \beta_{c, 1}+\beta_{T}\right)
$$

and therefore an appropriate ordering for the Hadamard codes is one which minimizes the number of crossing bands. Since the crossing bands are associated with a sign transition in the Hadamard code [see (44b)], an appropriate ordering of the codes is in ascending order of the number of sign transitions.

\section{REFERENCES}

[1] "In the matter of revision of Part 15 of the Commission's rules regarding ultra-wideband transmission systems,", FCC Rep. 02-48, Apr. 2002.

[2] Handbook of Global Optimization, R. Horst and P. M. Pardalos, Eds., Kluwer, Dordrecht, The Netherlands, 1995.

[3] J. W. Adams and J. L. Sullivan, "Peak-constrained least-squares optimization," IEEE Trans. Signal Process., vol. 46, no. 2, pp. 306-321, Feb. 1998.

[4] B. Alkire and L. Vandenberghe, "Convex optimization problems involving finite autocorrelation sequences," Math. Program. A, vol. 93, no. 3, pp. 331-359, Dec. 2002.

[5] A. Batra et al., "Multi-band OFDM physical layer proposal for IEEE 802.15 Task Group 3a," IEEE P802.15 Working Group for WPANs, IEEE Doc. P802.15-03/268r3, Mar. 2004.

[6] S. Boyd and L. Vandenberghe, Convex Optimization. Cambridge, U.K.: Cambridge Univ. Press, 2004.

[7] X. Chen and T. W. Parks, "Design of FIR filters in the complex domain," IEEE Trans. Acoust., Speech, Signal Process., vol. ASSP-35, pp. 114-153, Feb. 1987.

[8] J. O. Coleman and D. P. Scholnik, "Design of nonlinear-phase FIR filters with second-order cone programming," in Proc. 1999 Midwest Symp. Circuits Systems, vol. 1, Aug. 1999, pp. 409-412.

[9] T. N. Davidson, "Efficient design of waveforms for robust amplitude modulation," IEEE Trans. Signal Process., vol. 49, no. 12, pp. 3098-3111, Dec. 2001.

[10] T. N. Davidson, Z.-Q. Luo, and J. F. Sturm, "Linear matrix inequality formulation of spectral mask constraints with applications to FIR filter design," IEEE Trans. Signal Process., vol. 50, no. 11, pp. 2702-2715, Nov. 2002.

[11] T. N. Davidson, Z.-Q. Luo, and K. M. Wong, "Design of orthogonal pulse shapers for communications via semidefinite programming," IEEE Trans. Signal Process., vol. 48, no. 5, pp. 1433-1445, May 2000.

[12] T. N. T. Goodman, C. A. Micchelli, G. Rodriguez, and S. Seatzu, "Spectral factorization of Laurent polynomials," Adv. Comput. Math., vol. 7, no. 4, pp. 429-454, 1997.

[13] X. Luo, L. Yang, and G. B. Giannakis, "Designing optimal pulse-shapers for UWB radios," J. Commun. Netw., vol. 5, no. 4, pp. 344-353, Dec. 2003.

[14] J. H. McClellan and T. W. Parks, "A unified approach to the design of optimum FIR linear-phase digital filters," IEEE Trans. Circuit Theory, vol. CT-20, pp. 697-701, Nov. 1973.

[15] P. Moulin, M. Anitescu, K. O. Kortanek, and F. A. Potra, "The role of linear semi-infinite programming in signal-adapted QMF bank design," IEEE Trans. Signal Process., vol. 49, no. 9, pp. 2160-2174, Sep. 1997.

[16] S. Nordebo and Z. Zhang, "A unified approach to digital filter design with time- and frequency-domain specifications," IEEE Trans. Circuits Syst. II, Analog Digit. Signal Process., vol. 46, pp. 765-775, Jun. 1999.

[17] B. Parr, B. Cho, K. Wallace, and Z. Ding, "A novel UWB pulse design algorithm,” IEEE Commun. Lett., vol. 7, no. 5, pp. 219-221, May 2003. 
[18] J. G. Proakis, Digital Communications, 4th ed. New York: McGrawHill, Feb. 2001.

[19] S. Roy, J. R. Foerster, V. S. Somayazulu, and D. G. Leeper, "Ultrawideband radio design: the promise of high-speed, short-range wireless connectivity," Proc. IEEE, vol. 92, pp. 295-311, Feb. 2004.

[20] R. A. Scholtz, "Multiple access with time hopping impulse modulation," in Proc. Military Communications Conf., Boston, MA, Oct. 1993, pp. 447-450.

[21] I. W. Selesnick, M. Lang, and C. S. Burrus, "A modified algorithm for constrained least square design of multiband FIR filters without specified transition bands," IEEE Trans. Signal Process., vol. 46, no. 2, pp. 497-501, Feb. 1998.

[22] J. F. Sturm, "Using SeDuMi 1.02, a MATLAB toolbox for optimization over symmetric cones," Optim. Meth. Software, vol. 11-12, pp. 625-653, 1999.

[23] L. Vandenberghe and S. Boyd, "Semidefinite programming," SIAM Rev., vol. 31, no. 1, pp. 49-95, Mar. 1996.

[24] M. Z. Win, "Spectral density of random UWB signals," IEEE Commun. Lett., vol. 6, pp. 526-528, Dec. 2002.

[25] M. Z. Win and R. A. Scholtz, "Impulse radio: How it works," IEEE Commun. Lett., vol. 2, pp. 36-38, Feb. 1998.

[26] S. Wu, S. Boyd, and L. Vandenberghe, "FIR filter design via spectral factorization and convex optimization," in Applied and Computational Control, Signals and Circuits, B. Datta, Ed. Boston, MA: Birkhauser, 1999, vol. 1.

[27] X. Wu, Z. Tian, T. N. Davidson, and G. B. Giannakis, "Optimal waveform design for UWB radios," in Proc. IEEE Int. Conf. Acoustics, Speech, Signal Processing (ICASSP), vol. 4, May 2004, pp. 521-524.

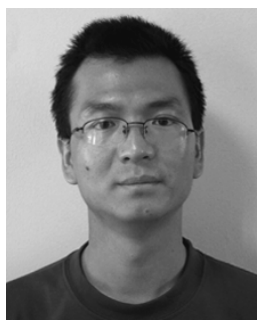

Xianren Wu (S'03) received the B.S. degree in communication engineering from Nanjing University of Posts and Telecommunications, Nanjing, China, in 1998 and the M.S. degree in information engineering from Beijing University of Posts and Telecommunications, Beijing, China, in 2001. From 2002 to 2005 , he was with the Michigan Technological University, Houghton.

His general research interests are wireless communications and coding theory.

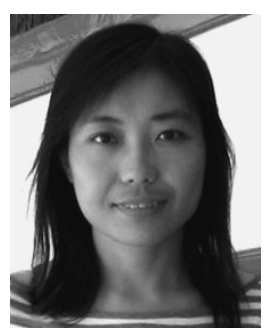

Zhi Tian (M'98) received the B.E. degree in electrical engineering from the University of Science and Technology of China, Hefei, China, in 1994 and the M.S. and Ph.D. degrees from George Mason University, Fairfax, VA, in 1998 and 2000, respectively.

From 1995 to 2000, she was with the Center of Excellence in Command, Control, Communications and Intelligence (C3I), George Mason University. Since 2000, she has been with the Department of Electrical and Computer Engineering, Michigan Technological University, where she is an Associate Professor. Her current research focuses on signal processing for wireless communications, particularly on ultra-wide-band systems.

Dr. Tian is an Associate Editor for IEEE TRANSACTIONS ON WIRELESS COMMUNICATIONS. She received a 2003 NSF CAREER award.

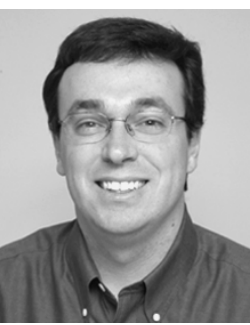

Timothy N. Davidson (M'96) received the B.Eng. (Hons. I) degree in electronic engineering from the University of Western Australia (UWA), Perth, in 1991 and the D.Phil. degree in engineering science from the University of Oxford, U.K., in 1995.

$\mathrm{He}$ is currently an Associate Professor in the Department of Electrical and Computer Engineering, McMaster University, Hamilton, ON, Canada, where he holds the (Tier II) Canada Research Chair in Communication Systems. His research interests are in signal processing, communications, and control, with current activity focused on signal processing for digital communication systems. He has held research positions with the Communications Research Laboratory, McMaster University; the Adaptive Signal Processing Laboratory, UWA; and the Australian Telecommunications Research Institute, Curtin University of Technology, Perth.

Dr. Davidson received the 1991 J. A. Wood Memorial Prize and the 1991 Rhodes Scholarship for Western Australia.

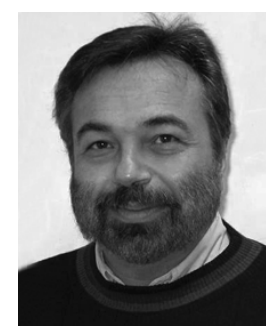

Georgios B. Giannakis (F'97) received the diploma in electrical engineering from the National Technical University of Athens, Greece, 1981. He received the M.Sc. and Ph.D. degrees in electrical engineering and the M.Sc. degree in mathematics from the University of Southern California (USC), Los Angeles, in 1983, 1986, and 1986, respectively.

After lecturing for one year at USC, he joined the University of Virginia in 1987, where he became a Professor of electrical engineering in 1997. Since 1999, he has been a Professor with the Department of Electrical and Computer Engineering, University of Minnesota, where he now holds an ADC Chair in Wireless Telecommunications. His genera interests span the areas of communications and signal processing, estimation and detection theory, time-series analysis, and system identification—subjects on which he has published more than 200 journal papers, 350 conference papers, and two edited books. His current research focuses on transmitter and receiver diversity techniques for single- and multiuser fading communication channels, complex-field and space-time coding, multicarrier, ultra-wideband wireless communication systems, cross-layer designs, and distributed sensor networks.

G. B. Giannakis is the co-recipient of six paper awards from the IEEE Signal Processing (SP) and Communications Societies (1992, 1998, 2000, 2001, 2003 , 2004). He also received the SP Society's Technical Achievement Award in 2000. He was Editor-in-Chief for the IEEE Signal Processing LeTTERS, Associate Editor for the IEEE TRANSACTIONS ON Signal PROCESSING, and the IEEE SigNAL PROCESSING LETTERS, Secretary of the SP Conference Board, a member of the SP Publications Board, a member and Vice-Chair of the Statistical Signal and Array Processing Technical Committee, Chair of the SP for Communications Technical Committee, and a member of the IEEE Fellows Election Committee. He was a member of the IEEE-SP Society's Board of Governors, the Editorial Board for the PROCEEDINGS OF THE IEEE, and the steering committee of the IEEE TRANSACTIONS ON WIRELESS COMMUNICATIONS. 